\title{
The weak-line T Tauri star V410 Tau
}

\section{A flaring star}

\author{
M. Fernández ${ }^{1}$, B. Stelzer ${ }^{2}$, A. Henden ${ }^{3}$, K. Grankin ${ }^{4}$, J. F. Gameiro ${ }^{5,6}$, V. M. Costa ${ }^{5,7}$, \\ E. Guenther ${ }^{8}$, P. J. Amado ${ }^{1}$, and E. Rodriguez ${ }^{1}$
}

\author{
${ }^{1}$ Instituto de Astrofísica de Andalucía, CSIC, Camino Bajo de Huétor 24, 18008 Granada, Spain \\ e-mail: matilde@iaa.es \\ 2 INAF - Osservatorio Astronomico di Palermo, Piazza del Parlamento 1, 90134 Palermo, Italy \\ 3 USRA/USNO Flagstaff Station, PO Box 1149, Flagstaff, AZ 86002-1149, USA \\ ${ }^{4}$ Ulug Beg Astronomical Institute, Astronomicheskaya 33, 700052 Tashkent, Uzbekistan \\ 5 Centro de Astrofisica da Universidade do Porto, Rua das Estrelas, 4150 Porto, Portugal \\ ${ }^{6}$ Departamento de Matemática Aplicada, Faculdade de Cíencas da Universidade do Porto, 4169 Porto, Portugal \\ 7 Departamento de Matemática, Instituto Superior de Engenharia do Porto, 4150 Porto, Portugal \\ 8 Thüringer Landessternwarte, Karl-Schwarzschild-Observatorium, Sternwarte 5, 07778 Tautenburg, Germany
}

Received 7 October 2003 / Accepted 3 July 2004

\begin{abstract}
We show that V410 Tau, a weak-line T Tauri star, is a flaring star. This result comes from an intensive, coordinated monitoring campaign carried out in November 2001 at visible and X-ray wavelength ranges. It is confirmed by previous, isolated observations found in the literature. Flares tend to occur mainly around the star's minimum brightness, when the most active regions face us.

We report on the strongest flare detected up to now on this star, for which we have obtained simultaneous visible Strömgren photometry and intermediate resolution spectroscopy. We derive decay times from 3 to $0.7 \mathrm{~h}$ at several wavelengths for the continuum in the 3600-5600 Å range. We estimate the energy involved in this and the other flares for which we have good time sampling, and conclude that the strongest event, at least, could have important consequences for the matter in the surroundings of the star. If similar events took place on the young Sun and lasted for several Myr, they could explain the anomalous abundances of elemental isotopes found in some meteorites. They could have also contributed to eliminate part of the primary atmospheres of the planet embryos and would have provided enough energy for the melting of solid iron-magnesium silicates, a process that may explain the presence of chondrules in chondritic meteorites.

High resolution spectroscopy of the $\mathrm{H}_{\alpha}$ emission line in the quiescent states of V410 Tau enables us to study the variability of the broad component. We suggest that this component is related to microflaring activity, such as the one observed on more evolved, magnetically-active stars. The large velocities and the energy associated with this component support this hypothesis.
\end{abstract}

Key words. stars: pre-main sequence - stars: individual: V410 Tau - stars: flare - stars: formation

\section{Introduction}

A spectrum taken on December 24, 1960 by Metreveli (1966) showed the Balmer and $\mathrm{Ca}$ II lines in emission in the K3-K4V star $\mathrm{BD}+28^{\circ} 637$ (later called V410 Tau) and it was, therefore, classified as a suspected variable star. These lines were not observed again in emission in the other spectra that $\mathrm{M}$. Metreveli took between 1956 and 1964, but photographic photometry performed in the 1960s confirmed the star as a variable (Mosidze 1970). Observations carried out in the following two decades focused only on the brightness variability of the star.

The lack of both strong emission lines and infrared excess ${ }^{1}$ was used to classify this star as a weak-line T Tauri star. This

\footnotetext{
${ }^{1}$ Its small infrared excess is attributed to one, maybe two, close companions at sub-arcsecond separation (Ghez et al. 1993; Ghez et al. 1997).
}

term has been coined to distinguish a sub-class of low mass (below $2 M_{\odot}$ ) pre-main sequence stars from the also very young classical T Tauri stars, for which the inner parts of an accretion disk are responsible for ultraviolet and infrared excesses, as well as a moderate to strong, variable emission line spectrum which gets superimposed on the photospheric one. For V410 Tau the origin of the variability is thought to be no longer associated with an accretion disk, because the mass accretion process has already finished, but to strong magnetic activity, an enhanced version of what is observed in the Sun.

Magnetic activity seems to explain all the phenomena observed on this star, setting off variabilities with a large range of timescales: from the long term, and sometimes irregular, changes of its amplitude over the years, to the $\mathrm{H}_{\alpha}$ variability with timescales of months, and the rotationally-modulated 
continuum light curve, which has a period of 1.871970 days $^{2}$ (Stelzer et al. 2003, hereafter Paper I, see their Introduction for more details about V410 Tau). Although the strength of the magnetic field itself has not been measured (the photospheric lines are too broad, due to the high rotational velocity), there is direct evidence for it from spectropolarimetric observations (Donati et al. 1997).

The photometric monitoring programmes carried out on V410 Tau up to now have only rarely shown variability on the shortest timescale (hours), which might be attributed to flares. Flares are defined as sudden, short timescale increases in the brightness of a star. They are believed to be due to a release of energy accumulated in the magnetic field, as it suddenly changes to a more stable configuration through a process called magnetic reconnection. In visible light, flares appear in the Sun as patches of intensely bright and white light (Carrington 1860) close to dark spots or spot groups.

The first stellar flare reported in the literature was discovered accidentally by Hertzsprung in 1924 on DH Car. In 1953 Haro and his collaborators started a programme at the observatory of Tonanzintla (Mexico) whose results, together with contributions from other observatories, led to the discovery of a great number of flares on young stars. In the following two decades more than 300 flares were discovered in Orion. Studies carried out in the Pleiades, NGC 2264, Praesepe and other young stellar clusters and associations clearly revealed that every star goes through a phase of flare activity in an early stage of its life (Gurzadyan 1980).

$\mathrm{T}$ Tauri stars are well known to show flaring activity, but the probability of detecting one of these events is quite low. In a review of flares on T Tauri stars, Gahm (1986) concluded that the fraction of time during which a star changes its ultraviolet flux by more than $20 \%$ within $3 \mathrm{~h}$ is 0.03 . This fraction is smaller if the variation threshold increases (Gahm 1990). This percentage was later confirmed by Gahm et al. (1995) and it shows that the flare frequency follows a power law, which is affected by the technique employed and, therefore, the sensitivity, as indicated by the flare rates observed spectroscopically or in the X-ray range. Following the analysis of 2320 spectra of weak-line $\mathrm{T}$ Tauri stars during a flare monitoring programme, Guenther \& Ball (1999) found a Balmer emission line flare frequency of $\sim 0.06 h^{-1}$. This slightly higher value, compared to that of Gahm et al. (1995), is most probably due to the high sensitivity of the Balmer lines to flares. The frequency of X-ray flares for $\mathrm{T}$ Tauri stars has been studied by Stelzer et al. (2000) from a systematic search carried out on the Taurus-Auriga-Perseus sky region using ROSAT PSPC observations. They found that these stars were in a flare state during $0.86 \pm 0.16 \%$ of the time.

Since the line enhancement observed in 1960 for V410 Tau, two flares have been reported in the continuum visible light for this star (Rydgren \& Vrba 1983; Vrba et al. 1988) and were observed mainly in the $U$ band. All other flares have been detected by means of spectroscopic lines, which turn into emission during such events (Hatzes 1995; Welty \& Ramsey 1995;

\footnotetext{
2 This is the period representative of the main spot that dominates the light curve observed.
}

Guenther \& Ball 1999), except for the radio flare observed by Cohen et al. (1982) at $6 \mathrm{~cm}$ wavelength, which was recognized as a flare due to the low brightness level of all subsequent radio observations (Bieging et al. 1984). No X-ray flares have been observed on V410 Tau in ROSAT observations, although its count rate is variable on timescales from months to years (Costa et al. 2000). We have also checked the ultraviolet IUE spectra in the INES Archive Data Server ${ }^{3}$ and they show no signs of flaring activity.

In order to analyze the connection between the regions (photosphere, chromosphere and corona) involved in the stellar magnetic activity of V410 Tau, we organized a visible and $\mathrm{X}$-ray observing campaign in 2001. Photometric, and intermediate and high resolution spectroscopic observations were planned to coincide with three Chandra satellite pointings scheduled for the visible light curve minimum and maximum levels. The photometric monitoring was carried out using the Johnson $U B V R_{\mathrm{J}}$, Johnson-Cousins $U B V(R I)_{\mathrm{c}}$, and the Strömgren $u v b y$ photometric systems in order to cover a wide wavelength range and, simultaneously, to have a good sampling in the blue region (with the aim of studying a possible scatter in the $U$ band). During the 11-day monitoring campaign several flares were observed, and one of them was very strong. A detailed analysis of both the rotationally modulated light curve and the X-ray observations was presented in Paper I. Here we discuss in detail the spectroscopic results and the flare activity on V410 Tau.

\section{Observations and data reduction}

Simultaneous visible and X-ray observations were planned for V410 Tau from Nov. 15-Nov. 26 UT, 2001. Visible photometry and spectroscopy was carried out from several observatories, while three X-ray observations were scheduled with Chandra using the Advanced CCD Imaging Spectrometer for Spectroscopy (ACIS-S).

A detailed description of the observations and data reduction procedure was given in Paper I. For the analysis and discussion in this current paper, only the photometric sampling intervals remain to be described. The Strömgren photometry carried out at the Sierra Nevada Observatory (Spain) was organized in such a way that a set of simultaneous $u v b y$ measurements of V410 Tau took place every $7 \mathrm{~min}$, with an integration time of 1 min on the target; two comparison stars and their three corresponding background skies were measured between consecutive measurements. At Mount Maidanak Observatory (Uzbekistan) the time required for an $U B V R_{\mathrm{J}}$ measurement varied between 3 and $4 \mathrm{~min}$, this time interval being occasionally longer. A set of $U B V R_{\mathrm{c}} I_{\mathrm{c}}$ measurements was taken at the USNO, Flagstaff Station (NOFS), every $20 \mathrm{~min}$.

Medium resolution spectroscopy was done at the Sierra Nevada Observatory during most of the strongest flare. The full width half maximum $(F W H M)$ of the lines of the calibration lamps was $1.7 \AA$ for the blue (4000-5160 $\AA$ ) and $1.5 \AA$ for the red (5645-6790 $\AA$ ) wavelength ranges. Spectroscopic observations in the blue range began $\sim 32$ min after the flare

\footnotetext{
${ }^{3}$ http://ines.laeff.esa.es/
} 


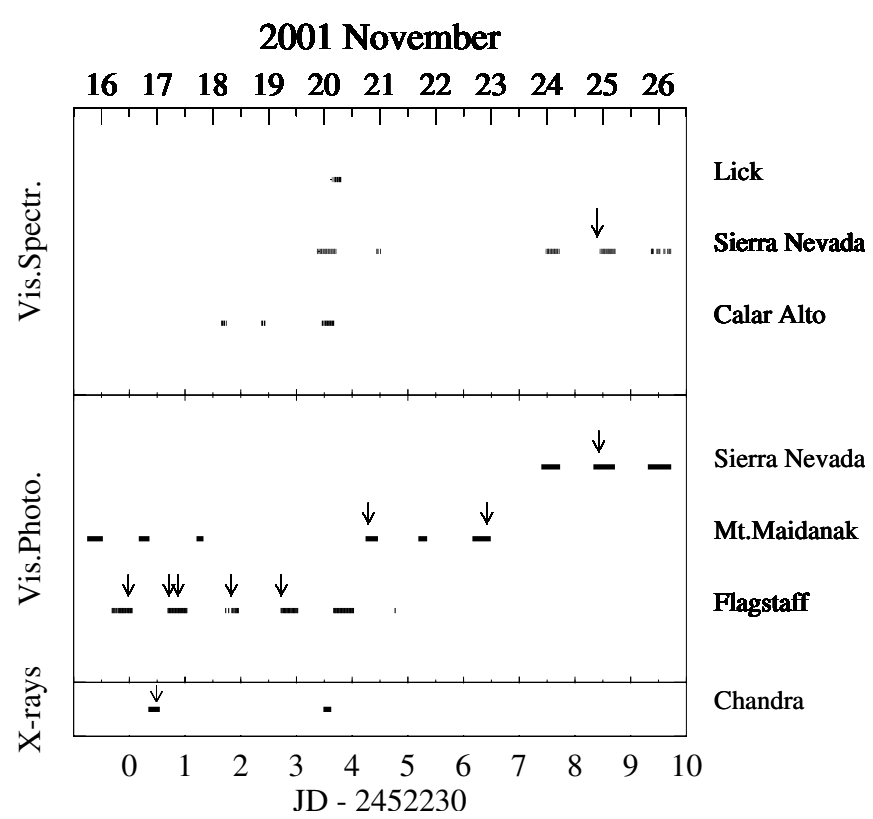

Fig. 1. Observing journal for November 2001. The occurrence of flares on V410 Tau is marked with vertical arrows. Note that our campaign included a third Chandra observation which was performed in March 2002 and is not shown in this diagram.

peak due to technical problems, and the first red spectrum was taken $2 \mathrm{~h}$ after the flare peak. The time interval between consecutive spectra was approximately $11 \mathrm{~min}$. Spectra were taken more frequently in the blue wavelength range.

The data reduction and analysis was done with IRAF (Image Reduction and Analysis Facility ${ }^{4}$ ).

\section{Flares on V410 Tau}

\subsection{Photometric monitoring}

In addition to the smooth variability due to star spots, V410 Tau reveals short-term variability which carries the clear signature of flares, i.e. intensity enhancements with short rise time and slower decay. Through a careful inspection of the light curves obtained during our campaign, a total of 8 flares have been identified, mainly in the $U$ and $B$ bands, plus a tentative one in X-rays. Figure 1 shows the occurrence of flares during our monitoring.

In order to study the characteristics of these phenomena we temporarily removed the flares from the data set, fitted the remaining photometric data in each band by a polynomial, and subtracted the fitted curve from the full data set (now including the flares). This way we eliminated variations due to the 1.87 day cycle. The remaining data allows to recover the evolution of the flare emission, and provides information about temperature changes (traced by colour variations), flare duration, and emitted energy. Figures 2 and 3 present a close up of the

${ }^{4}$ IRAF is distributed by the National Optical Astronomy Observatories, which is operated by the Association of Universities for Research in Astronomy, Inc. (AURA) under cooperative agreement with the National Science Foundation.
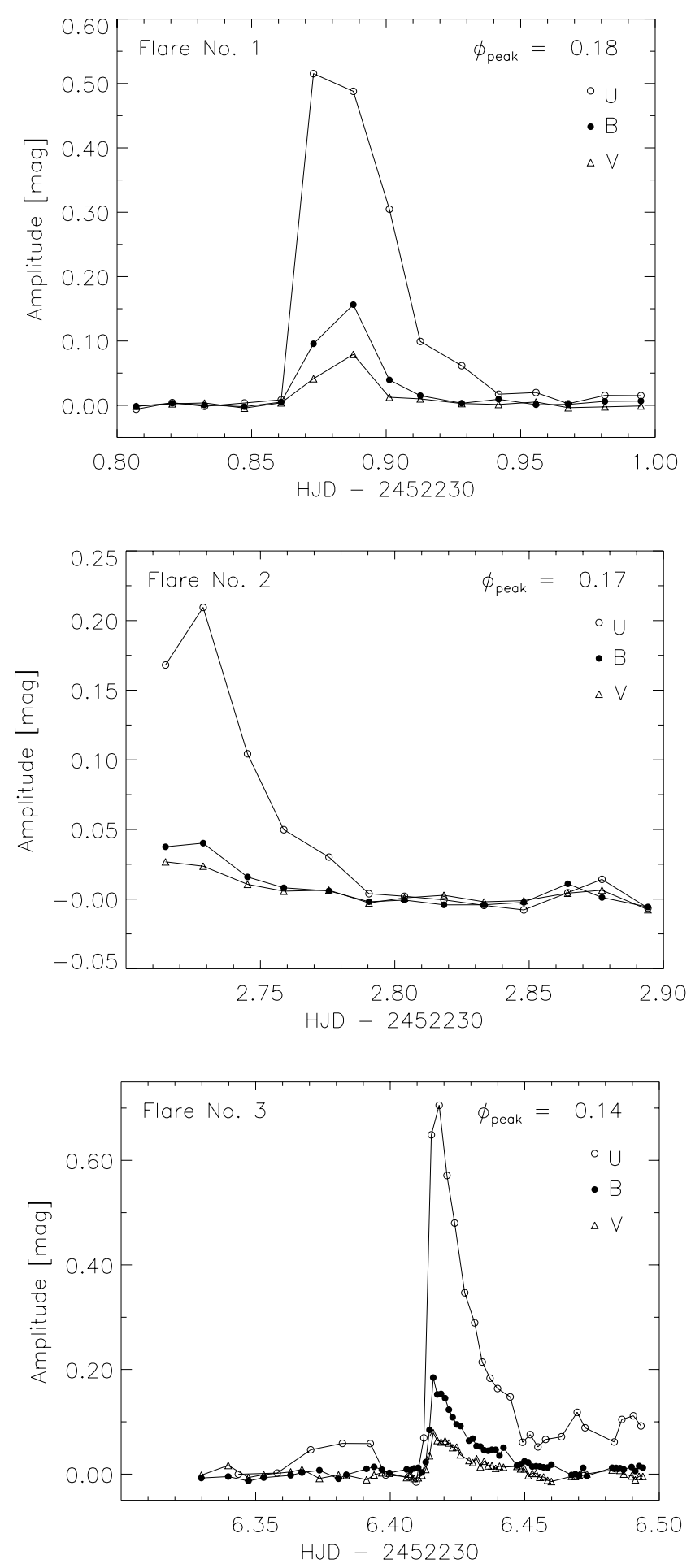

Fig. 2. Light curves of three of the four largest flares observed on V410 Tau in November 2001. The 1.87 day modulation of the light curve has been subtracted from the data in order to emphasize the evolution of the intensity change due to the flares. The scale of the time axis is the same for all panels.

photometry for the four largest flares observed in November 2001. In all cases the bulk of the flare emissions was restricted to shorter wavelengths, with none of them being detected in the $R_{\mathrm{J}}, R_{\mathrm{c}}$ or $I_{\mathrm{c}}$ bands. This is common in flares on young, late type stars (Haro et al. 1960; de Jager et al. 1986; Stepanov 1995) 
Table 1. Characteristic parameters for three of the four largest photometric flares observed on V410 Tau in November 2001. They were detected with the Johnson $U B V$ filters. The flare amplitudes, luminosities and energies refer to the maximum emission. The quiescent luminosity refers to the average luminosity just before the flare and was very similar for all three flares.

\begin{tabular}{lcccccccccccc}
\hline \hline & \multicolumn{3}{c}{ Flare amplitude (mag) } & \multicolumn{3}{c}{ Flare lum. $\left(\mathrm{erg} \mathrm{s}^{-1}\right)\left[\times 10^{32}\right]$} & \multicolumn{3}{c}{ Flare energy $(\mathrm{erg})\left[\times 10^{35}\right]$} & \multicolumn{4}{c}{ Decay time* (h) } \\
& $U$ & $B$ & $V$ & $U$ & $B$ & $V$ & $U$ & $B$ & $V$ & $U$ & $B$ & $V$ \\
\hline Flare \#1 & 0.51 & 0.15 & 0.08 & 1.17 & 1.32 & 1.24 & 4.21 & 2.97 & 2.38 & 1.5 & 1.1 & 0.9 \\
Flare \#2 & 0.19 & 0.03 & 0.02 & 0.38 & 0.14 & 0.07 & 1.4 & $0.4:$ & $0.2:$ & 1.6 & 1.1 & - \\
Flare \#3 & 0.77 & 0.17 & 0.08 & 2.18 & 1.51 & 1.25 & 1.71 & 1.33 & 1.35 & 0.9 & 0.8 & 0.7 \\
\hline Quiescent lum. & \multicolumn{10}{c}{2.0} & 9.3 & 18.3 \\
\hline
\end{tabular}

* Decay time back to $10 \%$ of the peak magnitude.

Table 2. Characteristic parameters for the largest photometric flare observed on V410 Tau in November 2001 with the uvby Strömgren filters. The flare amplitudes, luminosities and energies refer to the maximum emission, while the quiescent luminosity refers to the average luminosity just before the flare.

\begin{tabular}{|c|c|c|c|c|c|c|c|c|c|c|c|c|c|c|c|c|}
\hline & \multicolumn{4}{|c|}{ Flare amplitude (mag) } & \multicolumn{4}{|c|}{ Flare lum. $\left(\mathrm{erg} \mathrm{s}^{-1}\right)\left[\times 10^{32}\right]$} & \multicolumn{4}{|c|}{ Flare energy $(\mathrm{erg})\left[\times 10^{35}\right]$} & \multicolumn{4}{|c|}{ Decay time* $(\mathrm{h})$} \\
\hline & $u$ & $v$ & $b$ & $y$ & $u$ & $v$ & $b$ & $y$ & $u$ & $v$ & $b$ & $y$ & $u$ & $v$ & $b$ & $y$ \\
\hline Flare \#4 & 3.01 & 2.02 & 1.32 & 0.96 & 31.0 & 32.2 & 33.3 & 29.4 & 93.7 & 78.0 & 71.3 & 69.6 & 3.5 & 2.4 & 1.8 & 1.7 \\
\hline Quiescent lum. ( $\mathrm{erg} \mathrm{s}^{-1}$ ) & & & & & 2.06 & 5.91 & 14.0 & 20.5 & & & & & & & & \\
\hline
\end{tabular}

* Decay time back to $10 \%$ of the peak magnitude.

and is the result of both the smaller contribution of the flares and the higher quiescent photospheric emission of cool stars at these longer wavelengths.

Decay times back to $10 \%$ of the peak magnitude range from 3.5 to $0.9 \mathrm{~h}$ in the $U$ or $u$ bands. The stronger the flare, the longer its duration. For any given flare, decay times are shorter at longer wavelengths (see Tables 1 and 2 for details). Flare \#3 was preceded by a smaller event which was seen only in the $U$ band. Furthermore, after an exponential decay phase, the emission remained at a level higher than that of the pre-flare state. The other flares fell smoothly back to the emission level observed prior to the flare.

We have computed the luminosity released during the flares and at the corresponding quiescent states. For this calculation we have assumed a distance of $136_{-30}^{+54} \mathrm{pc}$ for V410 Tau (Wichmann et al. 1998); errors in the distance could affect fluxes by up to a factor of almost 2 . In order to convert magnitudes into absolute fluxes, we used the table provided by Rydgren et al. (1984) for the Johnson filters, and by Gray (1998) for the Strömgren filters. The effective wavelengths of all the photometric filters used are given in Table 3. Errors for the flux calibration of the Strömgren photometric system are about 1\% (Gray 1998). Effective wavelengths are sensitive to the star's spectral type (Landolt-Börnstein 1982). However, the changes estimated from a $\mathrm{K} 5$ to an $\mathrm{A} 0$ star in the $V$ band are always below $1.3 \%$ and do not affect our conclusions. We have not corrected for extinction because its value is compatible with $A_{v}=0$ mag (Cohen \& Kuhi 1979). The flare and quiescent luminosities, as well as the flare amplitudes, of the four strongest events are given in Tables 1 and 2, together with the total energies released. For their calculation we have computed the luminosity at each point of the light curve and we have assumed for it a duration defined by half of the time distance to the adjacent data points.
Table 3. Effective wavelength of the filters used in the photometric monitoring programme.

\begin{tabular}{lc}
\hline \hline & $(\AA)$ \\
Band & $\lambda_{\text {eff }}$ \\
\hline$u$ & 3491 \\
$v$ & 4111 \\
$b$ & 4662 \\
$y$ & 5456 \\
\hline$U$ & 3600 \\
$B$ & 4400 \\
$V$ & 5500 \\
$R_{\mathrm{c}}$ & 6760 \\
$R_{\mathrm{J}}$ & 7000 \\
$I_{\mathrm{c}}$ & 7910 \\
\hline
\end{tabular}

The spectral energy distribution of the star changes noticeably as a result of a strong flare, as seen from Fig. 4a for flare \#4. We have fitted black bodies to the quiescent state and to the flare peak (quiescent star + flare): in the quiescent state the effective temperature of V410 Tau is reproduced with an error of $10 \%$, and for the flare peak a temperature of $6900 \mathrm{~K}$ is estimated. If the stellar contribution is subtracted, the spectral energy distribution of the flare can be fitted by a black body of $8400 \mathrm{~K}$ (Fig. 4 b). These black body temperatures, however, can only be taken as an indication of the high temperatures involved in the event, because it is unlikely that stellar flares emit black body spectra (van den Oord et al. 1996).

Flares \#1 and \#4 present almost flat spectral energy distributions associated with temperatures lower than those of flares \#3 and, especially, \#2. This fact results from the large differences between the $U$ and the $B$ and $V$ amplitudes for the latter two flares. 


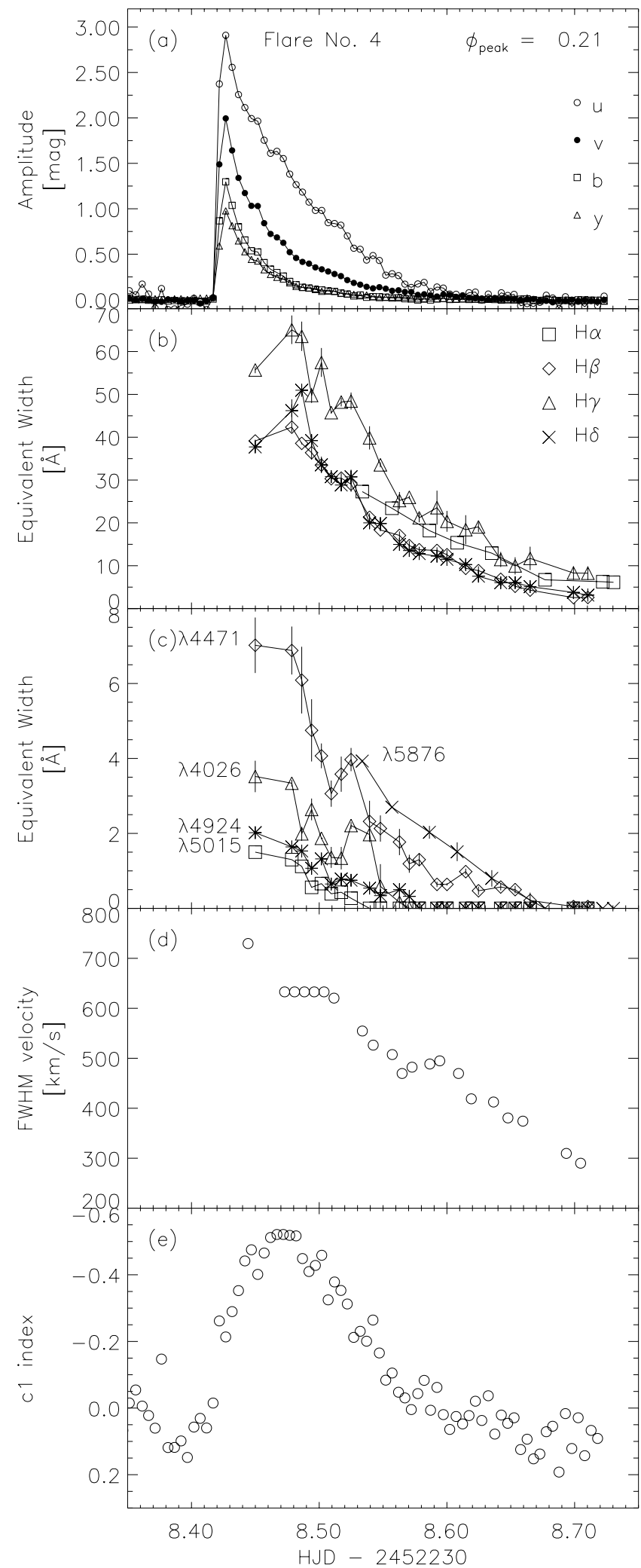

Fig. 3. Time evolution of different activity diagnostics during flare \#4, observed simultaneously with visible intermediate band photometry and spectroscopy. From top to bottom: a) light curves in Strömgren bands, b) equivalent widths of some Balmer lines, c) equivalent widths of the most prominent helium lines and the Fe II 4924 line, d) decrease of the full width half maximum velocity of the $\mathrm{H}_{\beta}$ emission, and e) time evolution of the Strömgren index c1.
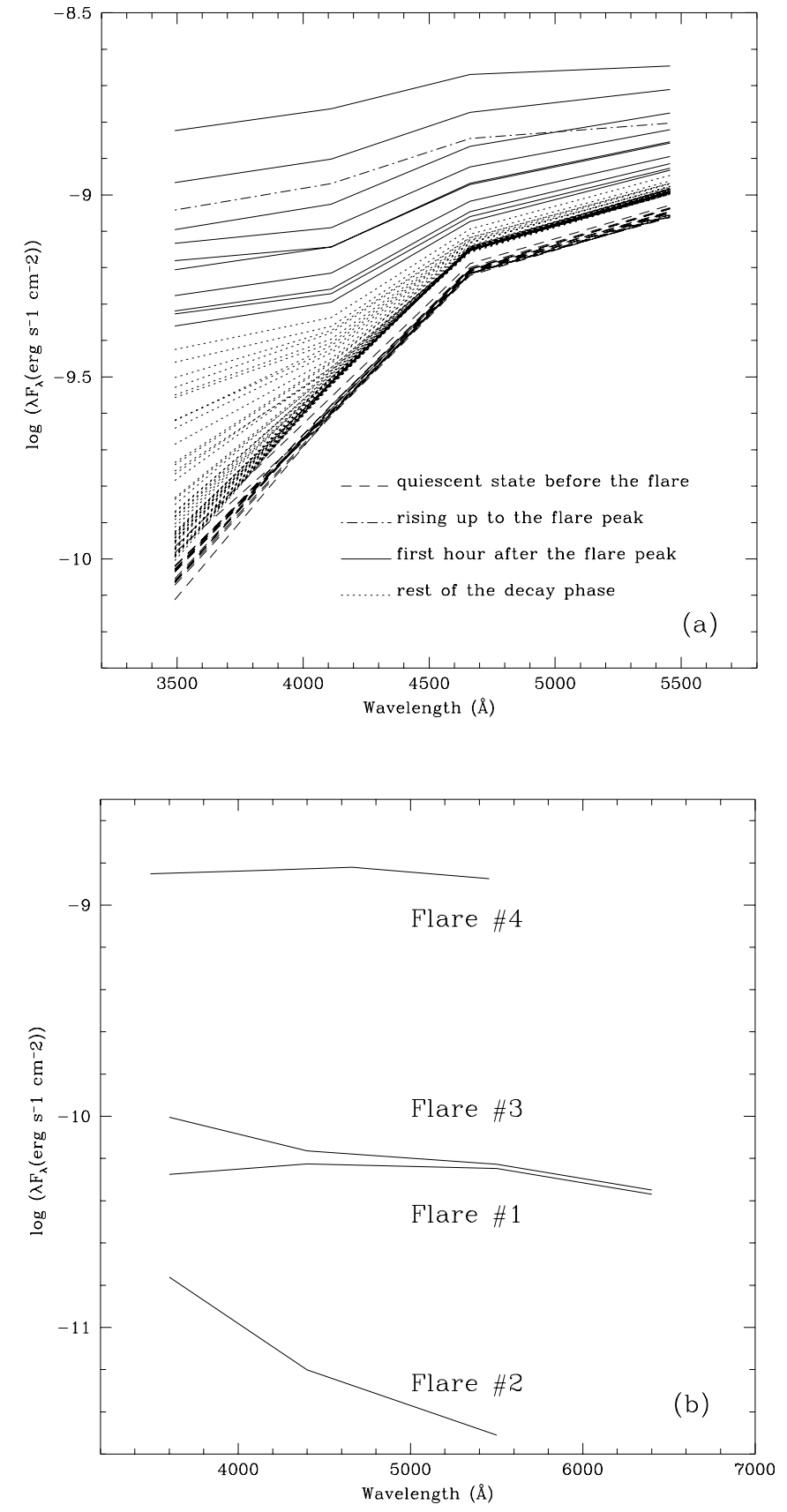

Fig. 4. a) Spectral energy distributions of V410 Tau during the flare \#4 rise and decay and in the quiescent stage (uvby observations). The diffence between the quiescent states before and after the flare is due to the spots. b) Spectral energy distributions of the four strongest flares, after subtracting the stellar contribution (UBVR observations).

\subsection{Spectroscopic signatures of flare \#4}

Flare \#4, the strongest event during our campaign, was also observed spectroscopically at intermediate resolution. During the flare a number of emission lines appeared, which are not seen in the quiescent spectrum: $\mathrm{H}_{\beta}, \mathrm{H}_{\gamma}, \mathrm{H}_{\delta}$, He II 4686, Fe II 4924, and the He I lines at $\lambda 4026 \AA, 4471 \AA, 5015 \AA$ and $5876 \AA$. They are identified on the spectrum shown in Fig. 5. We measured their equivalent widths in all the flare spectra and fitted their time evolution with an exponential (see panels b) and c) of 

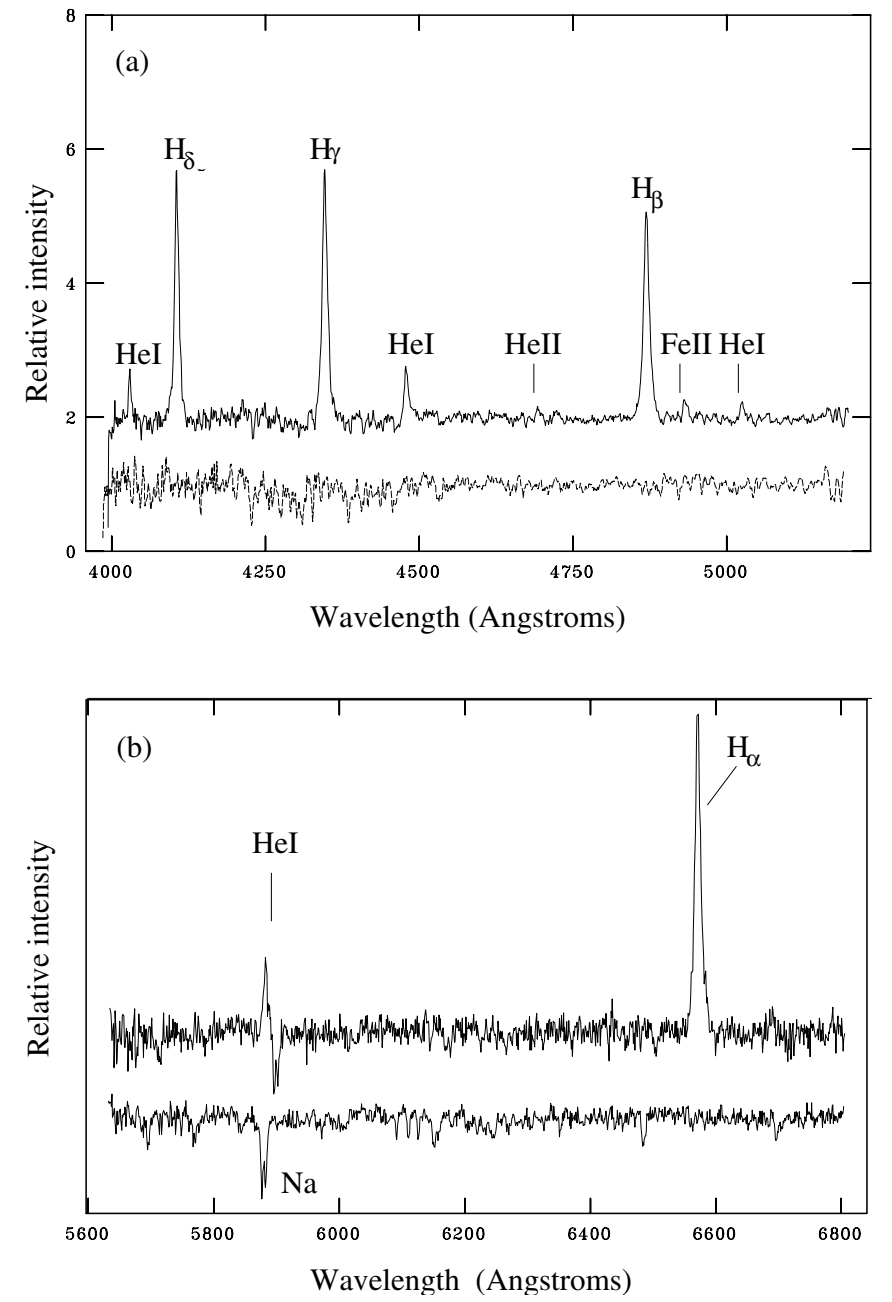

Fig. 5. Comparison between a) the blue and b) red spectra of flare \#4 and the average quiescent stage. In both panels the flare spectra have been shifted upwards for clarity.

Fig. 3). For $\mathrm{H}_{\gamma}$ and $\mathrm{H}_{\delta}$ the decay slows down after a first rapid decrease, and two exponentials are needed to provide a good fit. The HeI lines decay faster than the $\mathrm{H}$ lines, the latter being still in emission by the time both the HeI and the continuum light have returned to their quiescent levels.

The Balmer lines $\mathrm{H}_{\beta}, \mathrm{H}_{\gamma}$ and $\mathrm{H}_{\delta}$ show a broad emission, which gets narrower as the flare event decays and which can always be resolved at our intermediate spectral resolution. We have analyzed the decrease of the $F W H M$ of these lines. The behaviour is most obvious for $\mathrm{H}_{\beta}$, which decreases from a $F W H M$ of $730 \mathrm{~km} \mathrm{~s}^{-1}$, measured half an hour after the flare peak, to $290 \mathrm{~km} \mathrm{~s}^{-1}$ at the end of our measurements (see Fig. 3d). For flares on other stars some of the line broadening during flares has been attributed to the greatly increased gas density during the flare, while the rapidly varying broadenings, often asymmetric, are thought to be due to the fact that the flare produces rapid motions in the atmosphere of the star, with velocities of up to $1000 \mathrm{~km} \mathrm{~s}^{-1}$ (Byrne 1992).

Line fluxes are about $1 \%$ of the fluxes measured in the photometric Strömgren bands, and the values for the lines in the first spectrum taken after the flare peak are reported in Table 4. The difference in flux between higher and lower Balmer lines
(Balmer decrement) for this flare is clearly smaller than that found in classical $\mathrm{T}$ Tauri stars, as can easily be seen from the comparison between our spectrum (Fig. 5) and those of the classical T Tauri stars studied by the Valenti et al. (1993). Such a behaviour has also been observed in other flare stars, like the red dwarf EV Lac (Abdul-Aziz et al. 1995), for which the $\mathrm{H}_{\gamma} / \mathrm{H}_{\beta}$ ratio is even larger than for $\mathrm{V} 410 \mathrm{Tau}$.

\subsection{Balmer jump}

From the Strömgren photometry of flare \#4 we have computed the $m_{1}$ and $c_{1}$ indices $^{5}$. For $\mathrm{F}$ and $\mathrm{G}$ type stars, $m_{1}$ measures the line blanketing and $c_{1}$ the Balmer discontinuity (Golay 1974). It is remarkable that these indices reach their lowest values some time after the flare peak: $36 \mathrm{~min}$ for the $m_{1}$ index $\left(m_{1}=\right.$ $0.11 \mathrm{mag})$ and $57 \mathrm{~min}$ for the $c_{1}$ index $\left(c_{1}=-0.52 \mathrm{mag}\right)$. These minimum values are quite different from those expected for a $\mathrm{K} 3-\mathrm{K} 4 \mathrm{star}$, for which $m_{1} \sim 0.5 \mathrm{mag}$ and $c_{1}$ is between 0.3 and $0.5 \mathrm{mag}$ (Crawford \& Barnes 1970). In particular, the $c_{1}$ index must be affected by changes at the Balmer discontinuity, as suggested by the strong emission observed in the higher Balmer lines. We have compared this index with that of a typical classical T Tauri star with a similar spectral type (K5V), for which strong mass accretion has been proven, $\mathrm{LkH}_{\alpha} 264$. Values of $c_{1}$ in the range -0.77 to $-0.35 \mathrm{mag}$ have been reported for this star (Mendoza et al. 1990) and its spectrum clearly shows the Balmer jump in emission (Valenti et al. 1993). The $c_{1}$ index observed for V410 Tau might suggest the existence of regions with temperatures higher than those estimated by fitting a black body to the spectral energy distribution of the flare and in which the Balmer jump turns into emission.

Furthermore, we have compared the average values of some equivalent widths reported for $\mathrm{LkH}_{\alpha} 264$ for the Balmer and HeI 5876 lines (Lago \& Gameiro 1998) with the first measurements of these lines carried out on V410 Tau during flare \#4 (about $32 \mathrm{~min}$ after the peak for the blue lines and $2 \mathrm{~h}$ after the peak for the red ones). These values are tabulated in Table 4. We have found differences in both the $\mathrm{HeI} 5876$ to $\mathrm{H}_{\alpha}$ ratio and the Balmer decrement. Those differences should enable the identification of such a flare on a classical $\mathrm{T}$ Tauri star with weak to moderate lines, but would make it more difficult for those with strong lines in which the flare contribution is similar to that of the accretion process. The steep rise and the slow decay of flares seems to be a more accurate way to distinguish them from the more or less round-shaped accretion events. Two more relevant differences between a flare and a short time scale mass accretion event are: i) the rate at which the amplitude changes versus wavelength. This rate can be so steep that flares showing large amplitudes in the $U$ band can be accompanied by no detectable changes in the $R$ and $I$ bands (see Sect. 3.1); and ii) the line polarization, which has been measured in $\mathrm{H}_{\alpha}$ and $\mathrm{H}_{\beta}$ to be linearly polarized up to a small percentage during two solar flares (Hénoux \& Karlický 2003) and circularly polarized for the HeI 5878 emission line of BP Tau, a line which is associated with matter in the accretion columns (Johns-Krull et al. 1999).

\footnotetext{
${ }^{5} m_{1}=(v-b)-(b-y)$ and $c_{1}=(u-v)-(v-b)$.
} 
Table 4. Equivalent widths of the most prominent lines detected during flare \#4. A comparison is done with the classical T Tauri star $\mathrm{LkH}_{\alpha} 264$.

\begin{tabular}{lr|rr|rrr}
\hline \hline Star & & $\mathrm{H}_{\alpha}$ & HeI 5875 & $\mathrm{H}_{\beta}$ & $\mathrm{H}_{\gamma}$ & $\mathrm{H}_{\delta}$ \\
\hline V410 Tau & $E W(\AA)$ at JD 2 452 238.4443 & & & 39.1 & 55.7 & 37.7 \\
& Lum. $\left(\mathrm{erg} \mathrm{s}^{-1}\right)$ at JD 2 452 238.4443 & & & $2.0 \times 10^{31}$ & $2.9 \times 10^{31}$ & $1.5 \times 10^{31}$ \\
& $E W(\AA)$ at JD 2 452 238.5281 & 27.3 & 3.9 & & & \\
& Lum. $\left(\mathrm{erg} \mathrm{s}^{-1}\right)$ at JD 2 452 238.5281 & $1.4 \times 10^{31}$ & $1.2 \times 10^{30}$ & & & \\
$\mathrm{LkH}_{\alpha} 264$ & Average $E W(\AA)$ & 94.0 & 2.8 & 29.6 & 16.2 & 11.1 \\
\hline
\end{tabular}

\subsection{Flare frequency in November 2001}

A careful inspection of the $U B V$ light curves gathered throughout the campaign allowed us to find further intensity enhancements displaying a characteristic flare pattern. The total number of flares detected within our 11-day monitoring campaign is 9 , taking into account the brightness enhancement observed with Chandra and discussed in Paper I. Since the duration of the photometric monitoring after removing data gaps is $\sim 4.6$ days, the flare frequency during our observing campaign is about 2 per day. This is similar to what has already been found for other weak-line T Tauri stars (Guenther \& Ball 1999) and for the highly active, but more evolved, pre-main sequence star AB Dor (Vilhu et al. 1993).

The time resolution of our photometry varies between $\sim 4$ and $20 \mathrm{~min}$. It is thus shorter than the typical duration of a flare, providing good sensitivity for their detection. Nevertheless, the most important reason why we have detected so many flares seems to be that observations have been carried out at the $U$ and $B$ bands. Both amplitude and decay time are dramatically reduced when observing at longer wavelengths. Further support in this direction is given by the work of G.Haro and his collaborators (e.g., Haro \& Chavira 1969a,b) who detected numerous flare stars in Orion and in the Pleiades in the $U$ band using photographic plates. They, however, failed to detect a definite case of a flare star using hypersensitized infrared plates (Haro et al. 1960).

In the upper right corner of each panel in Fig. 2 we give the phase of the 1.87 day cycle corresponding to the peak of each flare in the $U$ band. All phases reported in this paper have been calculated using the new ephemeris from Paper I. Remarkably, the four strongest flares occurred at $\phi \sim 0.2$ at consecutive cycles (we might have missed one at JD 2452 234.6, not covered with our monitoring), indicating the presence of an emitting region which was active over at least eight days. The phases of another 3 flares are also located near the brightness minimum, suggesting a connection with the active regions responsible for the periodic 1.87 day variability. The remaining two flares occurred at maximum brightness, but they are by far the weakest ones we detected, with amplitudes in the $U$ band slightly above our detection limit. This accumulation of flares at certain phases on the folded light curve also suggests a small size for the flare loops. If the loops were much larger than the stellar radius, they would not be eclipsed as the star rotates and they should also be observable when the footpoints lie on the rear side of the star. This might explain the weakness of the two flares observed at maximum stellar brightness, since we might have seen only a small region of the flare.
Small sizes for flare loops are also supported by the most recent X-ray observations of coronal loops. Early estimates assuming radiatively cooling plasma suggested coronal loops on the order of, or even larger than, the stellar radius (Montmerle et al. 1983). For such large loops rotational modulation should be observed, and has been proven successful in explaining the peculiar shape of several flare light curves of late-type stars (Stelzer et al. 1999). Schmitt \& Favata (1999), however, observed an eclipsed flare on Algol, from which they derived the first direct evidence of a small X-ray loop. Other techniques, such as e.g. the comparison between the decay of X-ray light curves and hydrodynamic flare models (Stelzer et al. 2002), or X-ray line analysis (Ness et al. 2001), have also yielded loop sizes which are much smaller than the stellar radius.

The small loops that we propose for V410 Tau would connect active regions located not far from each other (on the stellar surface scale) and would fit very well in the context of a large spot being considered as an active region composed by numerous, smaller cool spots. Such a scenario is supported by the results obtained using the Doppler imaging technique with a CLEAN-like approach, which is highly efficient in determining the positions and shapes of star spots (Kürster 1993), and has been successfully applied to AB Dor (Kürster et al. 1993). In the case of this star, a group of small spots located in a certain region on the stellar surface is able to reproduce the photospheric absorption line distortions observed. The large spot suggested as the cause of the periodic light curve on V410 Tau, and which can be seen on the Doppler images, could very well be of this multi-spot nature.

\section{Microflaring on V410 Tau}

\subsection{The quiescent $H_{\alpha}$ line}

High resolution spectra, covering a wide wavelength range ( 3500-10000 $\AA$ ), show that on the entire quiescent spectrum of V410 Tau only the $\mathrm{H}_{\alpha}$ line appears in emission and presents clear variations in the intensity and shape of the profile. In order to study the variations of this line, we have subtracted the spectrum of a template star (rotationally broadened to a $v \sin i$ of $74 \mathrm{~km} \mathrm{~s}^{-1}$ ) from that of V410 Tau. This way can remove all photospheric lines that appear in the spectral range observed.

In Fig. 6 we show the sequence of $\mathrm{H}_{\alpha}$ residual profiles obtained at the Calar Alto and Lick observatories. We must point out that, for phases larger than 0.7 , the spectra have been obtained in poor weather conditions. As a consequence, they display a lower signal-to-noise ratio and strong telluric lines. For the very high resolution observations, like those obtained 


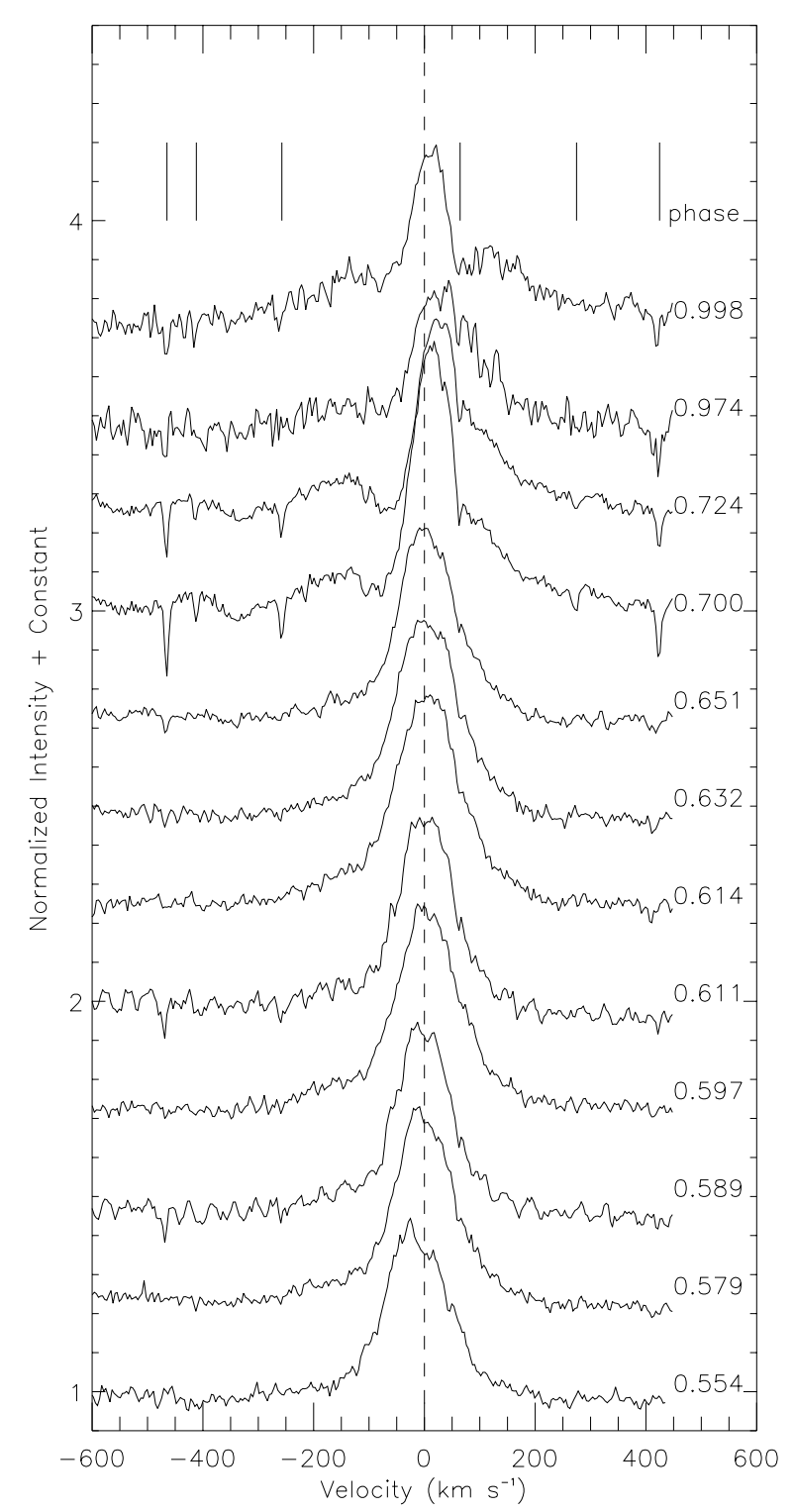

Fig. 6. Variations of $\mathrm{H}_{\alpha}$ residual profiles with phase. Residual profiles were obtained after subtracting a K2 template star. The intensity scale is given for the lower spectra and the rest are shifted in vertical scale for clarity. Vertical lines indicate telluric lines.

at the Lick observatory (at phases 0.700 and 0.724 ), the depth of the telluric lines is quite strong and the process of removing absorption lines is not as efficient as for the remaining spectra. Moreover, spectra taken close to the star's minimum brightness are better fitted by a K5 spectral type template, rather than the $\mathrm{K} 2$ type adopted throughout this work. This result cannot be simply due to the poor signal-to-noise ratio of the spectra, but to a mismatch in the spectral class due to the presence of cool spotted regions on the stellar surface.

The line and residual line equivalent widths measured are shown in Table 5. Uncertainties in the line measurements are due to the weak emission; the uncertainties associated with residual spectra are less than $10 \%$.

Changes in the profile shape can be seen in the residual $\mathrm{H}_{\alpha}$ line, as well as a shift towards the red in the central emission peak position from phases 0.55 to 1.0 . As shown in previous
Table 5. Radial velocities and $\mathrm{H}_{\alpha}$ equivalent widths (line and residual line) measured from the high resolution spectroscopic observations.

\begin{tabular}{ccccc}
\hline \hline HJD & Phase & $\begin{array}{c}V \mathrm{rad} \\
\left(\mathrm{km} \mathrm{s}^{-1}\right)\end{array}$ & \multicolumn{2}{c}{$E W\left(\mathrm{H}_{\alpha}\right)(\AA)$} \\
& & Line & Residual line \\
\hline 2452231.645 & 0.589 & $19.0 \pm 1.5$ & $1.08 \pm 0.11$ & $2.84 \pm 0.15$ \\
2452231.685 & 0.611 & $18.7 \pm 1.5$ & $1.09 \pm 0.10$ & $2.79 \pm 0.13$ \\
2452232.365 & 0.974 & $16.8 \pm 5.2$ & $0.68 \pm 0.12$ & $2.24 \pm 0.15$ \\
2452232.411 & 0.998 & $16.0 \pm 4.3$ & $0.61 \pm 0.10$ & $2.46 \pm 0.13$ \\
2452233.451 & 0.554 & $15.9 \pm 2.0$ & $1.20 \pm 0.12$ & $3.14 \pm 0.15$ \\
2452233.497 & 0.579 & $17.7 \pm 1.6$ & $1.19 \pm 0.09$ & $3.12 \pm 0.12$ \\
2452233.531 & 0.597 & $18.9 \pm 2.2$ & $1.19 \pm 0.09$ & $3.02 \pm 0.12$ \\
2452233.564 & 0.614 & $19.7 \pm 2.1$ & $1.21 \pm 0.12$ & $3.08 \pm 0.12$ \\
2452233.598 & 0.632 & $21.6 \pm 1.4$ & $1.16 \pm 0.10$ & $3.01 \pm 0.11$ \\
2452233.632 & 0.651 & $22.7 \pm 1.9$ & $1.02 \pm 0.09$ & $2.85 \pm 0.12$ \\
2452233.723 & 0.700 & $25.0 \pm 1.2$ & $0.59 \pm 0.10$ & $2.32 \pm 0.15$ \\
2452233.769 & 0.724 & $23.9 \pm 1.2$ & $0.04 * \pm 0.10$ & $1.89 \pm 0.12$ \\
\hline
\end{tabular}

* Strong blueshifted absorption.

works, there are two variable emission components in this line (e.g., Petrov et al. 1994; Hatzes 1995; Fernández \& Miranda 1998). Our observations show this pattern too, although more prominently in the residual profile where the two components are readily visible: a narrow emission peak close to the stellar rest velocity and a broad, less intense component producing extending wings (up to $\pm 300 \mathrm{~km} \mathrm{~s}^{-1}$ ). Superimposed on those two components is a blueshifted absorption feature that appears at some phases. The variability of the residual $\mathrm{H}_{\alpha}$ line shape seems to be related to the observation phase. On phases corresponding to the maximum brightness of the star, the residual $\mathrm{H}_{\alpha}$ profile is symmetrical. As the stellar brightness decreases, however, a blueshifted absorption develops, forming a P Cygni profile which might suggest the presence of a stellar wind.

In order to better characterize the general behaviour of the kinematic components, we have decomposed the $\mathrm{H}_{\alpha}$ line into the two abovementioned emission components by fitting a double Gaussian. When a clear absorption appeared, a third Gaussian in absorption was added. The fitting process is optimized by minimizing the $\chi^{2}$ statistic. The fit parameters are given in Table 6 and Fig. 7 shows the result of this procedure for two phases. The small number of spectra and the poor phase coverage do not allow a detailed analysis of the fit parameters. We are, however, convinced that, for the spectra obtained at phases greater than 0.7 , a third component in absorption is needed to improve the fit. This could explain the lack of strong variations in the residual $\mathrm{H}_{\alpha}$ equivalent widths between the high and low stellar brightness stages.

As for the velocity shift of both emission components, we found that the residual emission line shifts from the blue to the red, especially in the case of the narrow component (Fig. 8). The velocity shift is larger and negative at phases $\approx 0.6$, and increases reaching zero velocity at phases greater than 0.7 . This behaviour is similar to that of the absorption line velocity shifts. In the case of $\mathrm{H}_{\alpha}$, however, the narrow emission component is blueshifted while the absorption lines are redshifted. This is 
Table 6. Parameters of the Gaussian curves used for fitting the residual $\mathrm{H}_{\alpha}$ line. I: Gaussian maximum; V: Gaussian central velocity in km $\mathrm{s}^{-1}$; and $F W H M$ in $\mathrm{km} \mathrm{s}^{-1}$.

\begin{tabular}{ccccccccccc}
\hline \hline HJD & Phase & \multicolumn{3}{c}{ Component 1 } & \multicolumn{3}{c}{ Component 2 } & \multicolumn{3}{c}{ Component 3 } \\
& & \multicolumn{3}{c}{ Narrow } & \multicolumn{3}{c}{ Broad } & \multicolumn{3}{c}{ Absorption } \\
& & $I$ & $V$ & $F W H M$ & $I$ & $V$ & $F W H M$ & $I$ & $V$ & $F W H M$ \\
\hline 2452231.645 & 0.589 & 0.47 & -16 & 99 & 0.20 & -3 & 408 & - & - & - \\
2452231.685 & 0.611 & 0.47 & -13 & 101 & 0.20 & -5 & 364 & - & - & - \\
2452232.365 & 0.974 & 0.22 & 8 & 115 & 0.24 & 11 & 338 & -0.10 & -71 & 80 \\
2452232.411 & 0.998 & 0.29 & -4 & 62 & 0.26 & 9 & 374 & -0.04 & -90 & 44 \\
2452233.451 & 0.554 & 0.44 & -20 & 121 & 0.18 & 1 & 459 & - & - & - \\
2452233.497 & 0.579 & 0.46 & -17 & 102 & 0.21 & -4 & 367 & - & - & - \\
2452233.531 & 0.597 & 0.45 & -11 & 107 & 0.22 & -5 & 344 & - & - & - \\
2452233.564 & 0.614 & 0.46 & -10 & 113 & 0.21 & -1 & 344 & - & - & - \\
2452233.598 & 0.632 & 0.46 & -11 & 114 & 0.20 & 5 & 346 & - & - & - \\
2452233.632 & 0.651 & 0.45 & -11 & 100 & 0.21 & 7 & 338 & - & - & - \\
2452233.723 & 0.700 & 0.44 & -1 & 69 & 0.25 & 5 & 325 & -0.14 & -89 & 81 \\
2452233.769 & 0.724 & 0.32 & 4 & 71 & 0.25 & 5 & 305 & -0.21 & -65 & 101 \\
\hline
\end{tabular}
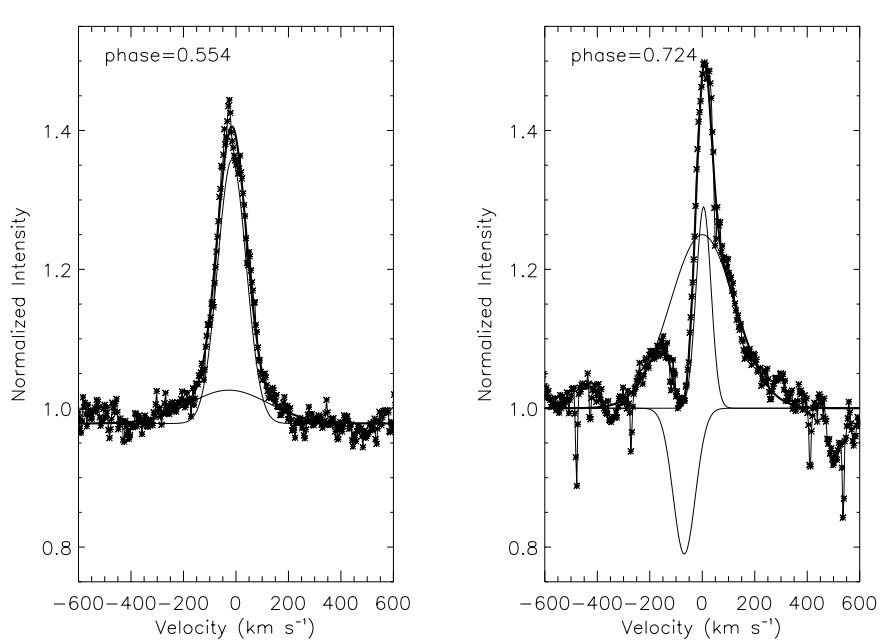

Fig. 7. Residual $\mathrm{H}_{\alpha}$ line profile decomposition. The thin lines show the individual components and the thick line represents the final fit.

what we would expect if the region responsible for the $\mathrm{H}_{\alpha}$ narrow component were related to the spots, which are responsible for the bumps on the absorption lines. In this scenario, when the largest group of spots becomes visible, a blueshifted $\mathrm{H}_{\alpha}$ emission shows up and the velocity shift decreases (in absolute value) as the spots move due to the stellar rotation. The fact that a velocity shift also occurs on phases near 0.6 means that the spot is visible at this phase, supporting the result shown by the Doppler images that the active region is quite large and near the pole.

\subsection{The $H_{\alpha}$ broad emission component}

A chromospheric origin has been suggested for the narrow peak; its velocity comes from the projected rotational velocity of the star at the position of the emitting regions. In the case of the broad component, the velocities involved reach more than $400 \mathrm{~km} \mathrm{~s}^{-1}$ and cannot be due the stellar rotation $(v \sin i$

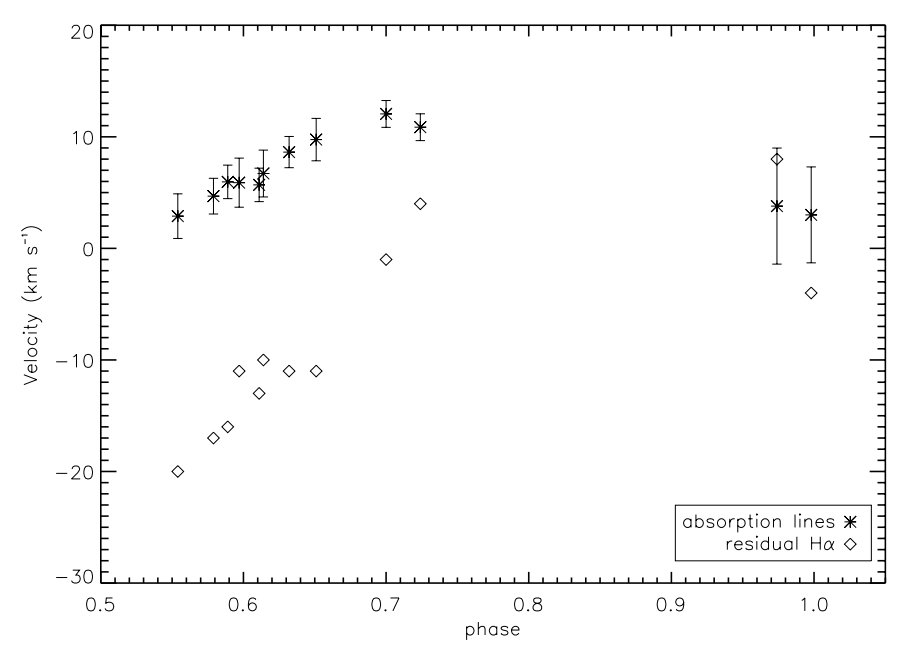

Fig. 8. Comparison between the radial velocity shift of the absorption lines and the velocity shift of the residual $\mathrm{H}_{\alpha}$ narrow component.

of $74 \pm 3 \mathrm{~km} \mathrm{~s}^{-1}$, Paper I). We also consider it quite improbable that it is due to the circumstellar gas environment or shell (Petrov et al. 1994), because V410 Tau shows very little infrared excess, and this excess has been attributed to one, maybe two, close companions at sub-arcsecond separation (Ghez et al. 1993; Ghez et al. 1997). Due to the strong evidence against the presence of circumstellar disk detectable remnant (Beckwith et al. 1990), we consider it more likely that the platform or extended wings are due to microflaring. This explanation has been provided by Montes et al. (1997, 1998) for the broad $\mathrm{H}_{\alpha}$ component of chromospherically active binary systems and weak-line T Tauri stars, and is supported by its similarity to the broad components found in the chromospheric $\mathrm{Mg}$ II h \& $\mathrm{k}$ lines, as well as in several transition region lines, of active stars. Linsky \& Wood (1994) have interpreted the broad component of the transition region lines as the result of microflaring, on the basis of the broad profiles observed in explosive events in the solar transition region. These events are 
thought to be associated with emerging magnetic flux regions where field reconnection occurs. An extensive description of the microflaring activity is given by Montes et al. (1997).

Our hypothesis of the broad component being due to microflaring is also supported by the position of V410 Tau in Fig. 21 of Montes et al. (1997). The left panel on their figure presents a correlation between the equivalent width of the $\mathrm{H}_{\alpha}$ broad component and that of the whole line for stars that show microflaring. On that diagram V410 Tau shares its position with one of these stars, II Peg.

For the strongest $\mathrm{H}_{\alpha}$ broad component detected $(\phi=0.724)$, we have estimated, using its equivalent width and the $R$ band brightness at that instant, that the energy emitted in this component is $8 \times 10^{29} \mathrm{erg} \mathrm{s}^{-1}$. This is about one order of magnitude below the smallest brightness variation that we are able to detect $(0.016 \mathrm{mag}$ in the $U$ band, which corresponds to $\left.3.9 \times 10^{30} \mathrm{erg} \mathrm{s}^{-1}\right)$. Thus, it seems reasonable to think that the broad $\mathrm{H}_{\alpha}$ emission comes from numerous weak flares that we are not able to detect photometrically. Time exposures for the high resolution spectra range from 45 to $60 \mathrm{~min}$. Robinson et al. (1999) reported microflaring activity on the dMe star YZCMi. They detected up to 54 flare events ranging in integrated flux from $2.0 \times 10^{28}$ to $3.0 \times 10^{30} \mathrm{erg}$ in the ultraviolet ( $2400 \AA$ ) during a 150 -min monitoring with the Hubble Space Telescope. If such a microflaring activity were taking place on V410 Tau, it could explain the broad $\mathrm{H}_{\alpha}$ component. Furthermore, if microflare events are important in the heating of stellar coronae, the higher X-ray luminosity of V410 Tau $\left(7.36 \times 10^{30} \mathrm{erg} \mathrm{s}^{-1}\right.$, Costa et al. 2000) compared to YZCMi, $L_{x}=3.0 \times 10^{28} \mathrm{erg} \mathrm{s}^{-1}$, would support a stronger microflaring activity on V410 Tau than on YZ CMi.

Flares are of central importance to coronal heating (Audard et al. 2001). Already in 1985, Doyle \& Butler and Skumanich drew attention to a correlation between X-ray luminosity and the time-averaged flare energy in the $U$ band on samples of flare stars, and they concluded that the quiescent coronal X-ray emission may be the result of heating from flare activity.

Fernández \& Miranda (1998) have reported noticeable changes in both the shape and maximum intensity of the $\mathrm{H}_{\alpha}$ emission, after comparing their spectra to others taken within less than one year (Petrov et al. 1994; Hatzes 1995). Since the $\mathrm{H}_{\alpha}$ equivalent widths in these spectra were always below a few $\AA$, flare activity can be discarded. In the light of a new explanation for the broad $\mathrm{H}_{\alpha}$ component as being due to microflares, variations in the strength of the microflaring activity could account for the variability observed. For example, there are notable changes between September 1993 and February 1994 on the broad $\mathrm{H}_{\alpha}$ component (Petrov et al. 1994; Hatzes 1995): the $\mathrm{H}_{\alpha}$ profile changed (at the phase of minimum brightness) from a strong narrow peak over a moderate broad component to a weak narrow emission in a profile dominated by a strong broad component. Two of these spectra also showed some HeI 5876 in emission, and since the $\mathrm{H}_{\alpha}$ equivalent width was never above $3.5 \AA$, the HeI emission could be related to peaks of the microflaring activity. Changes in the average level of this activity might have consequences on the heating of the corona and, therefore, on the X-ray luminosity of the star. This would also explain the lack of correlation that up to now has been found between the rotational period and the X-ray flux of V410 Tau, as well as the differences in X-ray luminosity that we found in Paper I in two Chandra measurements taken at similar phases, but several months apart.

Very weak flares, over one order of magnitude above the detected microflaring activity, could also explain the larger dispersion found in the V410 Tau $(U-B)$ color index at phases close to minimum brightness by Vrba et al. (1988). They noticed that the star does not get redder in this colour index at the minimum level of brightness; rather, it shows a concentration of blue values toward zero phase, when presumably the most active regions of the star face us. We are unable to check such a behaviour in our data because, after removing the flares, not enough data entries with a good signal-to-noise ratio remain at those phases.

Finally, residual emission is also found at the quiescent states for both $\mathrm{H}_{\beta}$ and the two Ca II lines $(\lambda 8498$, 18542) that we have observed. The $\mathrm{H}_{\beta}$ line peak shifts in the same way as that observed for $\mathrm{H}_{\alpha}$. The residual emission could be related to chromospheric and/or microflaring activity. Further research in this direction is needed.

\section{Discussion}

\subsection{Comparison with previous flares on V410 Tau}

We have gathered all the events considered as flares on this star in the literature and have compiled them in Table 7. All results come from visible photometric and spectroscopic observations, except for one radio flare observed by Cohen et al. (1982), during which the star increased its brightness at $6 \mathrm{~cm}$ wavelength by a factor of 14.5 above the average quiescent level observed by Bieging \& Cohen (1989).

In most of the occasions in which a flare was reported in the literature, very few data points (in some cases just one!) have been taken, except for two flare events detected by Welty \& Ramsey (1995) and Guenther \& Ball (1999). We have found a total of 12 possible flares. Their phases, which have been calculated using the ephemeris derived in Paper I, are tabulated in Table 7. If plotted on the phase folded light curve, flares tend to occur mainly in the region of the star's minimum brightness, i.e., they tend to gather at the phases when the spots, or at least the most active regions, are visible (Fig. 9). Only two weak flares have been detected at phases between 0.26 and 0.75 , when the star reaches its maximum brightness. In the case of the one detected by Guenther \& Ball (1999), the low value of the maximum equivalent width reported for $\mathrm{H}_{\beta}(0.6 \AA)$ points to a partially hidden flare or a very faint one. The second one, interpreted as a possible flare due to weak HeI 5876 emission (Hatzes 1995), might actually be an enhancement of the microflaring activity (see Sect. 4.2).

These results therefore confirm the suggestion of Vrba et al. (1988) that flares are located at the same longitudes as the most active regions (larger spots). Such a behaviour has also been reported for the Sun by Sammis et al. (2000), who have investigated the dependence of the occurrence of large flares on the magnetic structure of sunspots and conclude that almost all substantial flares occur in specific regions. Leto et al. (1997) 


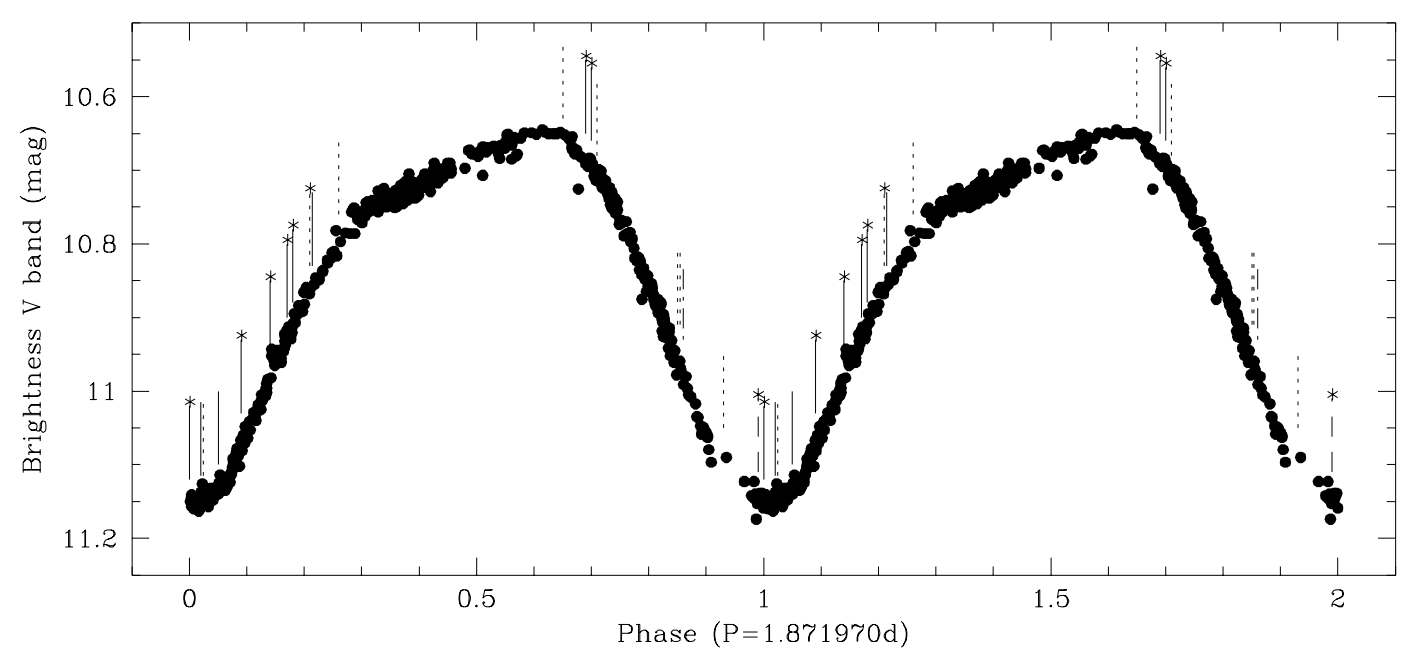

Fig. 9. Phase folded $V$ band light curve of all the observations carried out throughout our November 2001 monitoring campaign, after removing all the flares. On the light curve we indicate the distribution of flares and flare-like events on V410 Tau: nine flares identified during our monitoring in November 2001 (marked with asterisks), plus all flares previously reported in the literature (see Table 7). Different line types denote the technique used during the observation of each flare: visible photometry (solid), visible spectroscopy (dotted), X-ray (dashed) and radio (dash-dotted).

found a scenario for EV Lac that is reminiscent of the solar one because flares occurred more frequently in regions of emerging magnetic flux, i.e., where spots are developing.

Furthermore, many flares detected in November 2001 are located at those phases in which the edges (east-west) of the largest active region face us (Fig. 9). If we take into account what has been observed on the Sun, flares might be related to changes in the spots, more than to the spots themselves (Kiepenheuer 1953). Kiepenheuer also states that spots are sometimes accompanied by a revival of flare activity at the end of their visible life. In this context, we can interpret the numerous flares detected at phase $\phi \sim 0.2$ during November 2001 as changes on one edge of the largest group of spots. A careful inspection of the light curve before and after the flare would prove this hypothesis. Unfortunately, the strongest flare was observed at the end of our campaign. Therefore, further flare monitoring will be required to confirm this hypothesis. Such a change in the light curve has already been reported for II Peg, a RS CVn star, by Berdyugina et al. (1999). They found that the light curves before and after two months of strong flare activity are quite different. They computed two surface images derived from spectroscopic data which clearly show the break up of the large active region into two smaller ones.

Data from the literature also point to a long term variation of the flaring activity on V410 Tau, as shown by the fact that very similar monitorings carried out in different seasons came out with quite different results. The clearest example is given by Welty \& Ramsey (1995), who detected several weak flares during a 7-day monitoring campaign in December 1993, but had detected none during a previous 5-day observation carried out one year before, with almost the same instrumental setup. One month later, in January 1993, Fernández \& Miranda (1998) also failed to detect any flares during a 6-day spectroscopic observing run. Changes in the flaring activity levels have also been reported for other stars, e.g., EV Lac (Leto et al. 1997) or II Peg (Berdyugina et al. 1999).

\subsection{Flaring Balmer lines}

The flux ratios of the Balmer lines observed, their absolute fluxes, and the fact that flares are mainly observed close to minimum brightness are the ingredients for an analysis of the origin of the Balmer emission. As a starting point we use models of flares on other stars and then try to resolve the differences.

García-Alvarez et al. (2002) modelled flares on AT Mic with a slab model with an electron temperature of 13000 to $18000 \mathrm{~K}$, and an electron density of about $5.0 \times 10^{13}$ to $2.0 \times 10^{14} \mathrm{~cm}^{-3}$. Typical flare loops were assumed to have an effective thickness of about $2000 \mathrm{~km}$. In order to obtain some basic insights, we have carried out a parametric study using CLOUDY (Ferland 1996), a code for photoionization simulations. First of all, the fact that we find a stringent correlation between flare brightness and its distribution over the spot cycle implies not only that there is a correlation between spots and flares, but also that the flaring region is hidden by the star. This excludes all models in which the size of the loops is much larger than a stellar radius.

We first adopted a model similar to that of García-Alvarez et al. (2002) and assumed that the lines result from heating by the visible continuum emission observed. It turned out that it is impossible to reproduce the flux in $\mathrm{H}_{\beta}, \mathrm{H}_{\gamma}$ and $\mathrm{H}_{\delta}$, as well as the flux-ratios $\mathrm{H}_{\gamma} / \mathrm{H}_{\beta}$ and $\mathrm{H}_{\delta} / \mathrm{H}_{\beta}$, even if we assume that the flare covers a large fraction of the visible surface of the star. This is still true, even if we assume that matter is flowing. In this case we are able to reproduce the flux of, e.g., $\mathrm{H}_{\beta}$, but not the flux-ratios.

In the next step, we assumed that the matter that gives rise to the Balmer lines is heated by X-ray radiation. In this case, we find that the lower the temperature of the X-ray continuum, the better the agreement. It is unreasonable, however, to assume very low temperatures because X-ray observations imply temperatures of about $10 \mathrm{MK}$. 
Table 7. List with all historically recorded flares on V410 Tau, including the ones newly detected in our campaign. Note that, in some cases, the observations presented in the literature are merely indicative of a flare, by e.g. the deviation of one or a few data points from the usual behavior of V410 Tau, rather than by providing a convincing sampling of the time evolution of the flare.

\begin{tabular}{|c|c|c|c|c|}
\hline Date & JD & Phase & Band & Ref. \\
\hline \multicolumn{5}{|c|}{ Literature } \\
\hline Dec. 24,1960 & & & spect & 1 \\
\hline Mar. 22, 1981 & 2444686.2334 & 0.86 & radio & 2 \\
\hline Oct. 24,1981 & 2444901.8690 & 0.05 & photo & 3 \\
\hline Oct. 23,1985 & 2446361.951 & 0.02 & photo & 4 \\
\hline Dec. 3, 1993 & 2449324.9731 & 0.85 & spect & 6 \\
\hline Dec. 4,1993 & 2449325.6449 & 0.21 & spect & 6 \\
\hline Dec. 5, 1993 & 2449326.9893 & 0.93 & spect & 6 \\
\hline Dec. 6, 1993 & 2449327.5976 & 0.26 & spect & 6 \\
\hline Dec. 7, 1993 & 2449328.7143 & 0.85 & spect & 6 \\
\hline Dec. 1,1994 & 2449322.715 & 0.65 & spect & 5 \\
\hline Feb. 24, 1994 & 2449407.654 & 0.02 & spect & 5 \\
\hline Dec. 6, 1996 & 2450423.535 & 0.71 & spect & 7 \\
\hline \multicolumn{5}{|c|}{$\begin{array}{l}\text { This paper } \\
\end{array}$} \\
\hline Nov. 16, 2001 & 2452229.9842 & 0.70 & photo & \\
\hline Nov. 17, 2001 & 2452230.4870 & 0.97 & x-ray & \\
\hline Nov. 17, 2001 & 2452230.7110 & 0.09 & photo & \\
\hline Nov. 17, 2001 & 2452230.8730 & 0.18 & photo & Flare \#1 \\
\hline Nov. 18, 2001 & 2452231.8312 & 0.69 & photo & \\
\hline Nov. 19, 2001 & 2452232.7287 & 0.17 & photo & Flare \#2 \\
\hline Nov. 20, 2001 & 2452234.2918 & 0.00 & photo & \\
\hline Nov. 22, 2001 & 2452236.4160 & 0.14 & photo & Flare \#3 \\
\hline Nov. 24, 2001 & 2452238.4268 & 0.21 & $\mathrm{ph}+\mathrm{spec}$ & Flare \#4 \\
\hline
\end{tabular}

(1) - Metreveli (1966); (2) - Cohen et al. (1982); (3) - Rydgren \& Vrba (1983); (4) - Vrba et al. (1988); (5) - Hatzes (1995); (6) - Welty \& Ramsey (1995); (7) - Guenther \& Ball (1999).

As a third possibility, we tried out a model with an internal energy source. In the case of solar flares, it is well known that orders of magnitude more energy is released in plasma ejections and shock waves than in electromagnetic waves (Somov 1992). Possible heat sources are energetic electrons and heat flows. In this case we can easily reproduce the spectrum observed. While it is not possible to derive a unique solution, we can, for example, obtain a reasonable fit if we assume a loop that has a width, and a height, of $1 \%$ the stellar radius $(24000 \mathrm{~km})$, and a length of about a stellar radius. We further assume X-heating by a plasma of $10 \mathrm{MK}\left(10^{34} \mathrm{erg} \mathrm{s}^{-1}\right.$, which is the luminosity of flare \#4 in the visible wavelength range), as well as an internal heat source of about $1000 \mathrm{erg} \mathrm{s}^{-1} \mathrm{~cm}^{-3}$ and a flow speed of $400 \mathrm{~km} \mathrm{~s}^{-1}$. In this case, the values that we obtain for the logarithm of the flux $\left(\mathrm{erg} \mathrm{s}^{-1}\right)$ in $\mathrm{H}_{\beta}, \mathrm{H}_{\gamma}$ and $\mathrm{H}_{\delta}$ are 31.4, 31.4 and 31.4, respectively, which should be compared with the values that we observed: $31.3,31.4$ and 31.2 .

We thus conclude that the Balmer lines can only be reproduced if we assume an additional heat source, apart from heating by visible and $\mathrm{X}$-ray continuum radiation.

\subsection{Comparison with flares on other stars}

Although the term flare star was coined for dwarf stars of spectral class K and M (subclassified as UV Cet-type stars), Pettersen (1989) explored the presence of stellar flares across the HR diagram and found that recurrent flares occur in stars with sizable convection zones near or above the main sequence, and for both single and binary stars. Therefore this term includes, apart from the aforementioned UV Cet-type stars, young stars in star forming regions and open clusters, like Orion and the Pleiades; binary systems, like those of the RS CVn, BY Dra, Algol and W UMa type; and rapidly rotating FK Com-type single stars.

The largest solar flares can reach energies of the order of $E \sim 10^{32} \mathrm{erg}$, while the largest flares on dMe stars are of the order of $E \sim 10^{34}-10^{35} \mathrm{erg}$, and $E \sim 10^{36}-10^{38} \mathrm{erg}$ in the case of RS CVn stars (Haisch et al. 1991). Figure 10 shows the energy released in the $U B V$ bands during three strong flares that we detected on V410 Tau, as well as during the flares observed on a set of dMe flare stars from the literature (Kahler et al. 1982; Doyle et al. 1988, 1989; Jevremović et al. 1998). The strongest flare we observed on V410 Tau has been excluded from this figure because we did not observe it in the $U B V$ bands. The empirical relation derived by Lacy et al. (1976) based on flare data for UV Cet stars is also plotted. Gahm (1990) claimed that flares on $\mathrm{T}$ Tauri stars are found in the upper right part of this diagram with energies exceeding those of the bulk of UV Cet stars by 4-5 orders of magnitude. According to Gahm (1990), in the $E_{\mathrm{U}}$ versus $E_{\mathrm{B}}$ diagram the T Tauri stars lie below the Lacyrelation, on the dotted line in Fig. 10. Our data for V410 Tau confirm the extremely high energies, but we do not see a deviation from the $E_{\mathrm{U}}-E_{\mathrm{B}}$ relation found for flare stars. All flares of V410 Tau line up perfectly with the Lacy-relation and represent events of medium energy $\sim 10^{35}-10^{36} \mathrm{erg}$. The strongest flare we have detected on V410 Tau $\left(3 \times 10^{37} \mathrm{erg}\right)$ is among the most energetic ones found up to now in T Tauri stars (Gahm 1990), but it is surpassed by some flares detected on RS CVn stars.

Recursive flare events, like those we have observed for V410 Tau at phases $\sim 0.2$, have also been reported for a number of stars. García-Alvarez et al. (2003) detected, during a campaign devoted to flares on HR 1099, two active regions which flared during the same epoch, the flares showing a rotation periodicity which lasted for almost three consecutive orbits. Andrews et al. (1988) realized that all the flux enhancements that they observed on II Peg between 1981 and 1983 took place preferentially within a single hemisphere. The possibility of rather persistent, active longitudes in the photosphere is well known in the solar case, and García-Alvarez et al. (2003) have compiled information on such a phenomenon taking place in other flare stars too. There are also young stars that show periodic radio flaring, e.g., V773 Tau (Massi et al. 2002), but the origin of this periodicity seems to be quite different. In the case of V773 Tau, a binary T Tauri star, flares cluster at the periastron passage and have been attributed to recurrent interactions of giant loops, anchored on the two components. On the other hand, the quasi-periodic X-ray flares detected by Tsuboi et al. (2000) on the protostar YLW 15 seem to orginate in a star-disk interaction. 

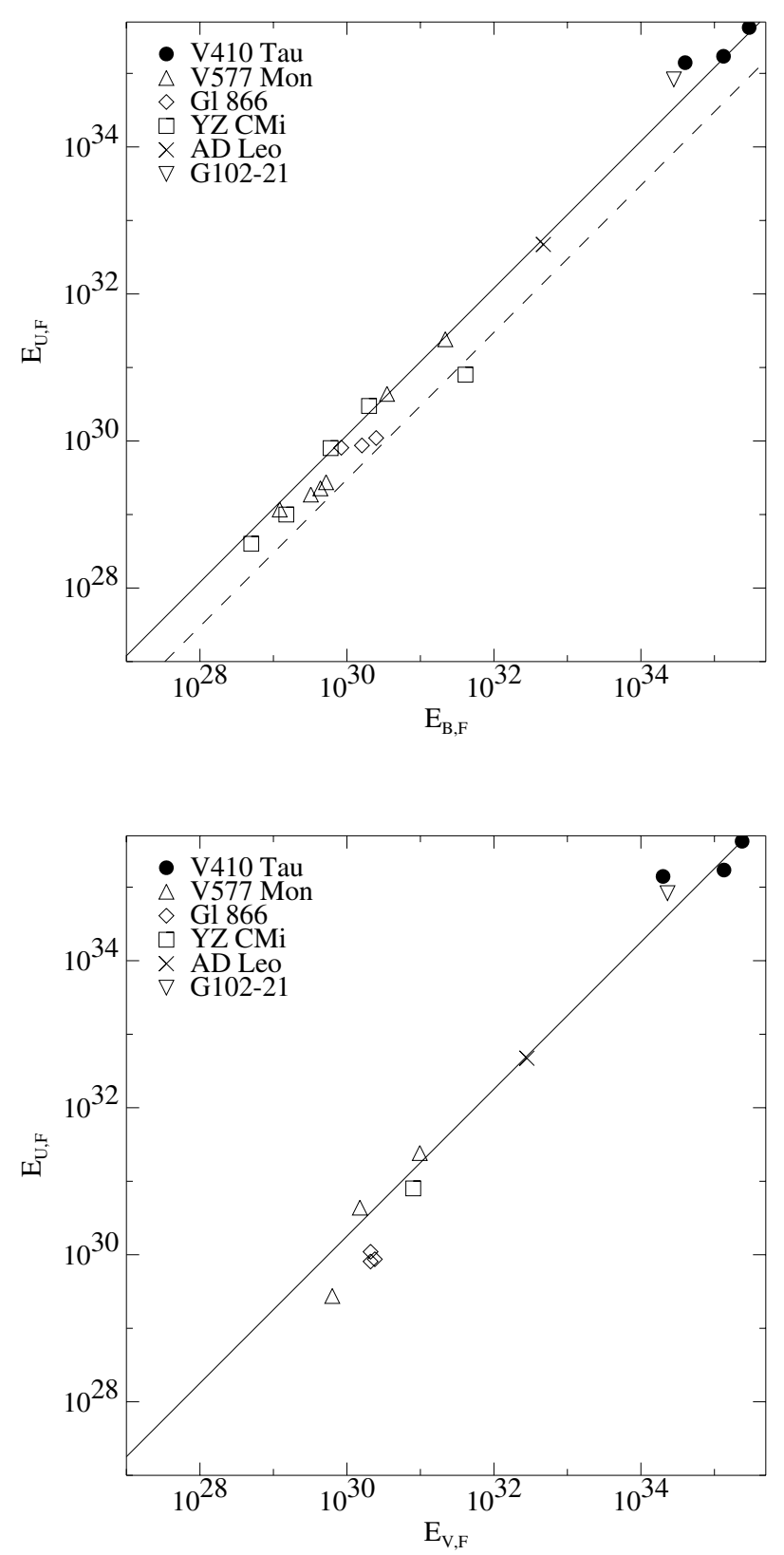

Fig. 10. Connection between the energies released in the $U B V$ bands during some flares observed on V410 Tau (flares \#1, \#2 and \#3) and on a sample of flare stars. The empirical relation derived by Lacy et al. (1976) based on flare data for UV Cet stars is plotted with a solid line. The T Tauri stars' locus, according to Gahm (1990), is shown by the dashed line.

\subsection{Effects of flares on young planetary systems}

The strength and frequency of flares on V410 Tau should not be a surprise if we take into account the strength of all the other phenomena related to the magnetic activity on this star. These flares might have important consequences on the surroundings of the star and, in order to evaluate these consequences, we need to know the amount of energy released at shorter (ultraviolet and soft X-ray) wavelengths. If the flares on V410 Tau behave in the way solar flares do, then similar amounts of energy are released in the visible, and the soft X-ray and ultraviolet ranges (Somov 1992). This is also the case, e.g., in a flare detected in the visible, radio and X-ray ranges on YZ CMi by Kahler et al. (1982). Its luminosity ratio in the soft X-ray and white light ranges averaged, over the entire event, $\sim 1.5$. The flares we have detected on V410 Tau would, therefore, indicate that energies of the order of $10^{37} \mathrm{erg}$ are released in the soft $\mathrm{X}$-ray and ultraviolet wavelength ranges.

Since V410 Tau can be considered as the Sun at one million years (Herbst 1989), if the flare frequency and strength observed last several Myr, they may explain some features observed in the Solar System, like i) the chondrules and the refractory inclusions in chondritic meteorites; ii) the anomalous abundances of elemental isotopes in chondrules and inclusions of the most pristine carbonaceous chondrites; and iii) the transition from primary atmospheres in the planetary embryos. Evidences for flares on older, post-T Tauri stars have been found during individual observations (e.g. BD-104662, Herbig 1977; HD 560, Tagliaferri et al. 1988; AB Dor, Pakull 1981) but no statistical results from large samples are yet available.

Chondrule precursors are thought to be loose balls of interstellar dust coagulated in the disk (Feigelson \& Montmerle 1999); but dust in the disk can only exist at certain, large enough, distances from the star. Muzerolle et al. (2003) have calculated the size of the dust-free gas disk for several classical $\mathrm{T}$ Tauri stars, and for those with K spectral type they give sizes of $\sim 0.1$ AU. On the other hand chondrule precursors can exist after the disk begins to dissipate (weak line $\mathrm{T}$ Tauri stars), because this dissipation only means that we are unable to detect either the gas or the warm dust. Bary et al. (2002) have reported the discovery of $\mathrm{H}_{2}$ gas orbiting a weak-line $\mathrm{T}$ Tauri star (DoAr 21), heretofore presumed nearly devoid of circumstellar material. They think that a significant amount of $\mathrm{H}_{2}$ remains in the gas phase, but only a tiny fraction of this mass emits in the near-infrared. Nevertheless, Mg-isotopic abundances in chondrules suggest that high total gas pressure prevailed during chondrule formation, even if it was in a very localized environment (Galy et al. 2000); to our best knowlegment it still has to be computed if such gas pressures are compatible with the pressure in the remaining disks around weak line $\mathrm{T}$ Tauri stars or in their flare eruptions.

All chemical fractionation trends observed in bulk chondritic meteorites require formation temperatures at around the condensation temperature of metallic iron or magnesian olivine: 1200-1400 K (Palme \& Boynton 1993). Some Ca, Al-rich inclusions (CAIs) in carbonaceous chondrites require formation temperatures as high as $1600 \mathrm{~K}$. As for the chondrules, there is evidence of their having been brought to melting temperatures of $2000 \mathrm{~K}$ (Jones et al. 2000; Shu et al. 2001). A review of the models proposed for the chondrule and CAIs formation is given by Jones et al. (2000). The models that are believed to be still viable are a) lightning in the protoplanetary nebula (although there are still some concerns about its feasibility); b) a shock wave, whose source(s) needs to be defined; and c) an X-wind that arises from the interaction between an accretion disk and a strongly magnetized central star (Shu et al. 2001). Nevertheless, the abovementioned dust-free disks found by Muzerolle et al. (2003) suggest that there may be difficulties for the X-wind model for chondrule formation. The energy 
released during the strongest event that we have detected on V410 Tau is probably enough to bring chondrules to melting temperatures $(\sim 2000 \mathrm{~K})$, thus providing an alternative scenario to the mentioned models.

In recent literature, the melting interval for chondrules has been restricted to less than a few minutes in order to facilitate the retention of moderately volatile elements such as $\mathrm{Na}$ and $\mathrm{S}$. Another constraint for this melting interval is the presence of relict grains that were not in contact with melt long enough to dissolve (see Jones et al. 2000, and references therein). Therefore, the maximum time available for substantial melting must have been from tens of seconds to several minutes. On the other hand, the preservation of chemically zoned mineral grains and the presence of glass indicate not only a rapid cooling, but also that the cooling continued down to low ambient temperatures, and that no significant reheating or annealing occurred (Connolly \& Hewins 1992; Jones \& Lofgren 1993; Jones et al. 2000). Both the short peak temperatures and the estimated cooling rates support the interpretation that chondrule formation was a fairly localized process (Jones et al. 2000). Strong flare events fulfill all these requirements and are also compatible with the fact that at least $25 \%$ of chondrules provide evidence of having experienced multiple heating events (e.g., Rubin \& Krot 1996).

As regards the second feature that the flare activity may explain: both the anomalous abundances of elemental isotopes in chondrules and the inclusions of the most pristine carbonaceous chondrites, as well as the high abundances of daughter products of some short-lived nuclides, all point to high-energy processes that occurred during the early stages of our Solar System (see, e.g., Feigelson et al. 2002; Gounelle et al. 2001). Although external phenomena, like supernova explosions, have been used to explain these abundances, it has been suggested that they could also be due to spallation reactions from energetic $(\mathrm{MeV})$ protons and ions originating in magnetic reconnection flares (see also Goswami et al. 2001; Marhas et al. 2002; Leya et al. 2003).

Finally, noticeable effects of flares like those observed on V410 Tau are also expected on larger, protoplanetary bodies. At an age of $1 \mathrm{Myr}$, Earth-like terrestrial planets (if formed according to the theory of the Earth formation) are still in their early stages. The Earth itself is supposed to have been formed after 50 or $100 \mathrm{Myr}$ (Taylor 1992) or in 10 to $100 \mathrm{Myr}$ (see references in Kasting 1993). Wetherill \& Stewart (1993) have estimated a time of about $0.1 \mathrm{Myr}$ for the accumulation of major fractions of terrestrial planets from the initial planetesimal stage, and recent calculations (Kokubo \& Ida 2000) show that in $0.5 \mathrm{Myr}$ protoplanets with masses of about $10^{26} \mathrm{~g}^{6}$ are formed at $1 \mathrm{AU}$. The abundances and isotopic patterns of surviving noble gases suggest a period during which a greatly enhanced ultraviolet flux drove a hydrodynamic escape flow of hydrogen (Hunten et al. 1991), which could have acted on a transient steam atmosphere that may have been formed during at least part of the accretionary period (Kasting 1993). This hydrodynamic escape can only exist if a large quantity of extreme ultraviolet energy is deposited in a region corresponding

\footnotetext{
${ }^{6}$ Earth mass: $5.976 \times 10^{27} \mathrm{~g}$.
}

to the ionosphere in a static atmosphere. Hunten et al. (1991) consider that the required flux is 100 times that of what is emitted by the present-day Sun. The four strongest flares that we have observed on V410 Tau are above this threshold. A further confirmation of the effect of flares on primary planetary atmospheres will require a further study of flares on both older weak-line $\mathrm{T}$ Tauri stars and post- $\mathrm{T}$ Tauri stars, covering a wide range of ages (from 5 to $\sim 60 \mathrm{Myr}$ ).

\section{Conclusions}

We have shown that V410 Tau is a flaring star and that flares tend to occur mainly close to the minimum brightness of the star, when its most active regions face us. This result comes out of an intensive, coordinated monitoring campaign in the visible and X-ray wavelength ranges carried out in November 2001, with the aim of studying correlations between the photometric spot cycle of V410 Tau and the chromospheric and coronal activity diagnostics. The flaring behaviour is confirmed by previous, isolated observations found in the literature. This compilation of measurements also points to long term variations in the level of flare activity.

Photometric observations at visible wavelengths were performed at three sites around the globe, thus providing complete phase coverage of the 1.87 day spot cycle, despite the short monitoring time, only 11 days. In the data obtained during our campaign 9 flares were identified. With a time resolution of $\sim 4$ to $\sim 20 \mathrm{~min}$ and a good signal-to-noise ratio in the $U$ band, our observations are very sensitive to the detection of flares, and we derive a flare rate of about two events per day.

Besides the strong flare activity detected during our campaign, we suggest microflaring for the quiescent stages, as observed through the broad $\mathrm{H}_{\alpha}$ component. This hypothesis is supported by i) the large velocities involved (the FWHM of this component ranging from 300 to over $400 \mathrm{~km} / \mathrm{h}$ ); and ii) the energy emitted in this component, which is about one order of magnitude below our detection limit in the photometry.

We discuss in detail the four strongest flares. Decay times range from 3.5 to $0.9 \mathrm{~h}$ at the $U$ or $u$ bands, and the energies released during the events total about $10^{35}-10^{36} \mathrm{erg}$, except for the strongest one, which reaches $3 \times 10^{37} \mathrm{erg}$. These energies are among the highest measured for flares on T Tauri stars, but they are surpassed by the strongest flares detected on RS CVn stars.

The energy released during the strongest event that we have detected is probably enough to bring chondrules to melting temperatures $(\sim 2000 \mathrm{~K})$, thus providing an alternative scenario to the protostellar flares in the fluctuating $\mathrm{X}$-wind model proposed by Shu et al. (2001), and the lightning or shock waves in the protoplanetary nebula. Flare events like the one we have observed could also explain the anomalous abundances of elemental isotopes in chondrules and the inclusions of the most pristine carbonaceous chondrites, and if they occur over several Myr, they might have important effects on the evolution of the primary atmospheres of Earth-like planets.

Acknowledgements. We want to acknowledge W.Herbst for producing and maintaining the $\mathrm{T}$ Tauri database at http:// www. astro.wesleyan.edu/ ${ }^{\text {bill/, }}$, and our two referees for their 
interesting and clarifying comments, which allowed us to improve the paper. Rafael Garrido and Andrés Moya are acknowledged for some preliminary observations made in 2000 which tested the two comparison stars used in our photometric OSN observations. Thomas Müller gave us some very interesting bibliography on chondritic meteorites, and Miguel Ángel López Valverde gave us very useful information on the atmospheres of planet embryos. With Enrique Pérez and Martin Kürster we shared very fruitful discussions and David Montes provided us with relevant information about microflaring. M.F. wants to acknowledge Jorge Fernández for his help while buying the computer with which most of the data from the Sierra Nevada Observatory have been reduced and analyzed, and María Fernández for improving the English of the paper; M.F. wants also to acknowledge the Thüringer Landessternwarte and the Max-PlanckInstitut für Extraterrestrische Physik in Garching for their hospitality. She was partially supported by the Spanish grant AYA2001-1696. B.S. acknowledges financial support from the European Union by the Marie Curie Fellowship Contract No. HPMD-CT-2000-00013. J.F.G. and V.C. were supported by grant POCTI/1999/FIS/34549 approved by FCT and POCTI, with funds from the European Community programme FEDER. P.J.A. acknowledges financial support at the Instituto de Astrofísica de Andalucía-CSIC by an I3P contract (I3P-PC2001-1) funded by the European Social Fund. This research has made use of NASA's Astrophysics Data System Bibliographic Services and was partly based on data obtained at the $90 \mathrm{~cm}$ and $1.5 \mathrm{~m}$ telescopes at the Sierra Nevada Observatory, which is operated by the Consejo Superior de Investigaciones Científicas through the Instituto de Astrofísica de Andalucía and at the GermanSpanish Astronomical Center, Calar Alto, which is jointly operated by the Max-Planck-Institut für Astronomie, Heidelberg, and the Instituto de Astrofísica de Andalucía (CSIC).

\section{References}

Abdul-Aziz, H., Abranin, E. P., Alekseev, et al. 1995, A\&AS, 114, 509

Andrews, A. D., Rodonó, M., Linsky, J. L., et al. 1988, A\&A, 204, 177

Audard, M., Güdel, M., \& Mewe, R. 2001 A\&A, 365, L318

Bary, J. S., Weintraub, D. A., \& Kastner, J. H. 2002, ApJ, 576, L73

Beckwith, S., Sargent, A., Chini, R. S., \& Güsten, R. 1990, AJ, 99, 924

Berdyugina, S. V., Ilyin, I., \& Tuominen, I. 1999, A\&A, 349, 863

Bieging, J. H., \& Cohen, M. 1989, AJ, 98, 1686

Bieging, J. H., \& Cohen, M., \& Schwartz, P. R. 1984, ApJ, 282, 699

Byrne, P. B. 1992, in The astronomy and astrophysics encyclopedia, ed. S. P. Maran (Cambridge; Cambridge University Press), 783

Carrington, R. C. 1860, MNRAS, XX, 13

Crawford, D. L., \& Barnes, J. V. 1970, AJ, 75, 978

Cohen, M., \& Kuhi, L. V. 1979, ApJS, 41, 743

Cohen, M., Bieging, J. H., \& Schwartz, P. R. 1982, ApJ, 253, 707

Connolly, H. C., Jr., \& Hewins, R. H. 1992, Lunar Planet. Sci., 23, 239

Costa, V. M., Lago, M. T. V. T., Norci, L., \& Meurs, E. J. A. 2000, A\&A, 354, 621

de Jager, C., Heise, J., Avgoloupis, S., et al. 1986, A\&A, 156, 95

Donati, J.-F., Semel, M., Carter, B.D., Rees, D. E., \& Cameron, A. C. 1997, MNRAS, 291, 658

Doyle, J. G., \& Butler, C. J. 1985, Nature, 313, 378

Doyle, J. G., Butler, C. J., Bryne, P. B., \& van den Oord, G. H. J. 1988, A\&A, 193, 229

Doyle, J. G., van den Oord, G. H. J., \& Butler, C. J. 1989, A\&A, 208, 208

Feigelson, E. D., \& Montmerle, T. 1999, ARA\&A, 37, 363
Feigelson, E. D., Garmire, G. P., \& Pravdo, S. H. 2002, ApJ, 572, 335

Ferland, G. J. 1996, Hazy, a Brief Introduction to Cloudy, University of Kentucky Department of Physics and Astronomy Internal Report

Fernández, M., \& Miranda, L. F. 1998, A\&A, 332, 629

Gahm, G. F. 1986, in Flare: Solar and Stellar, Rutherford Appleton Laboratory, RAL-86-085, 124

Gahm, G. F. 1990, in Flare Stars in Clusters, Associations and the Solar Vicinity, Proc. 137th IAU Symp. (Byurakan, Armenia: Kluwer Academic Publishers), 193

Gahm, G. F., Lodén, K., Gullbring, E., \& Hartstein, D. 1995, A\&A, 301, 89

Galy, A., Young, E. D., Ash, R. D., \& O’nions, R. K. 2000, Science, 290, 1751

García-Álvarez, D., Jevremović, D., Doyle, J. G., \& Butler, C. J. 2002, A\&A, 383, 548

García-Álvarez, D., Foing, B. H., Montes, D., et al. 2003, A\&A, 397, 285

Ghez, A. M., Neugebauer, G., \& Matthews, K. 1993, AJ, 106, 2005

Ghez, A. M., White, R. J., \& Simon, M. 1997, ApJ, 490, 353

Golay, M. 1974, Introduction to astronomical photometry (Dordrecht, Holland: D. Reidel Publishing Company), 182

Goswami, J. N., Marhas, K. K., \& Sahijpal, S. 2001, ApJ, 549, 1151

Gounelle, M., Shu, F. H., Shang, H., et al. 2001, ApJ, 548, 1051

Gray, R. O. 1998, AJ, 116, 482

Guenther, E. W., \& Ball, M. 1999, A\&A, 347, 508

Gurzadyan, G. A. 1980, Flare Stars (Pergamon Press Oxford), 199

Haro, G., \& Chavira, E. 1969a, Bol. Obs. Tonantzintla y Tacubaya, 5, 23

Haro, G., \& Chavira, E. 1969b, Bol. Obs. Tonantzintla y Tacubaya, 5, 59

Haro, G., Chavira, E., \& Mendoza, E. 1960, AJ, 65, 490

Hatzes, A. P. 1995, ApJ, 451, 784

Haisch, B., Strong, K. T., \& Rodonò, M. 1991, ARA\&A, 29, 275

Hénoux, J.-C., \& Karlický, M. 2003, A\&A, 407, 1103

Herbig, G. H. 1977, ApJ, 217, 693

Herbst, W. 1989, AJ, 98, 2268

Hunten, D. M., Gérard, J.-C., \& François, L. M. 1991, in The Sun in time, ed. C. P. Sonett, M. S. Giampapa, \& M. S. Matthews (Tucson: Univ. Arizona Press), 463

Jevremović, D., Butler, C. J., Drake, S. A., \& O’Donoghue, D., \& van Wyk, F. 1998, A\&A, 338, 1057

Johns-Krull, C. M., Valenti, J. A., Hatzes, A. P., \& Kanaan, A. 1999, ApJ, 510, L41

Jones, R. H., \& Lofgren, G. E. 1993, Meteoritics, 28, 213

Jones, R. H., Lee, T., Connolly, H. C., Jr., Love, S. G., \& Shang, H. 2000, Protostars and planets IV, ed. V. Mannings, A. P. Boss, \& S. S. Russell (The university of Arizona press), 927

Kahler, S., Golub, L., Harnden, F. R., et al. 1982, ApJ, 252, 239

Kasting, J. F. 1993, Science, 259, 920

Kürster, M. 1993, A\&A, 274, 851

Kürster, M., Schmitt, J. H. M. M., \& Cutispoto, G. 1993, A\&A, 289, 899

Kiepenheuer, K. O. 1953, in The Sun, ed. G. P. Kuiper (The University of Chicago Press), 380

Kokubo, E., \& Ida, S. 2000, Icarus, 143, 15

Lacy, C. H., Moffett, T. J., \& Evans, D. S. 1976, ApJS, 30, 85

Lago, M. T. V. T., \& Gameiro, J. F. 1998, MNRAS, 294, 272

Landolt-Börnstein, 1982, ed. K. Schaifers, \& H. H. Voigt (Heidelberg: Springer-Verlag), 2b, 81

Leya, I., Halliday, A. N., \& Wieler, R. 2003, ApJ, 594, 605

Leto, G., Pagano, I., Buemi, C. S., \& Rodonó, M. 1997, A\&A, 327, 1114 
Linsky, J. L., \& Wood, B. E. 1994, ApJ, 430, 342

Marhas, K. K., Goswami, J. N., \& Davis, A. M. 2002, Science, 298, 2182

Massi, M., Menten, K., \& Neidhöfer, J. 2002, A\&A, 382, 152

Mendoza, E. E., Rolland, A., \& Rodríguez, E. 1990, A\&AS, 84, 29

Metreveli, M. 1966, Astr. Circ. USSR No. 352, 3

Mosidze, L. N. 1970, Bull. Obs. Abastumani, 39, 21

Montes, D., Fernández-Figueroa, M. J., de Castro, E., \& Sanz-Forcada, J. 1997, A\&AS, 125, 263

Montes, D., Fernández-Figueroa, M. J., de Castro, E., et al. 1998, The Tenth Cambridge Workshop on Cool Stars, Stellar Systems and the Sun, ed. R. A. Donahaue, \& J. A. Bookbinder, ASP Conf. Ser., 154,1516

Montmerle, T., Koch-Miramond, L., Falgarone, E., \& Grindlay, J. E. 1983, ApJ, 269, 182

Muzerolle, J., Calvet, N., Hartmann, L., \& D’Alessio, P. 2003, ApJ, 597, L149

Ness, J.-U., Mewe, R., Schmitt, J. H. M. M., et al. 2001, A\&A, 367, 282

Pakull, M. W. 1981, A\&A, 104, 33

Palme, H., \& Boynton, W. V. 1993, Protostars and planets III, ed. E. H. Levy, \& J. I. Lunine (Tucson: The university of Arizona press), 979

Petrov, P. P., Shcherbakov, V. A., \& Berdyugina, S. V., et al. 1994, A\&AS, 107, 9

Pettersen, B. R. 1989, A\&A, 209, 279

Robinson, R. D., Carpenter, K. G., \& Percival, J. W. 1999, ApJ, 516, 916

Rubin, A. E., \& Krot, A. N. 1996, in Chondrules and the Protoplanetary Disk, ed. R. H. Hewins, R. H. Jones, \& E. R. D. Scott (Cambridge: Cambridge Univ. Press), 173
Rydgren, A. E., \& Vrba, F. J. 1983, ApJ, 267, 191

Rydgren, A. E., Schmelz, J. T., Zak, D. S., \& Vrba, F. J. 1984 (Publ. U.S.N.O.), Vol. XXV, Part I, 1

Sammis, I., Tang, F., \& Zirin, H. 2000, ApJ, 540, 583

Schmitt, J. H. M. M., \& Favata, F. 1999, Nature, 401, 44

Shu, F. H., Shang, H., Gounelle, M., Glassgold, A. E., \& Lee, T. 2001, ApJ, 548, 1029

Skumanich, A. 1985, Australian J. of Phys., 38, 971

Somov, B. V. 1992, Physical processes in solar flares (Dordrecht: Kluwer Academic Publishers)

Stelzer, B., Neuhäuser, R., Casanova, S., \& Montmerle, T. 1999, A\&A, 344, 154

Stelzer, B., Neuhäuser, R., \& Hambaryan, V. 2000, A\&A, 356, 949

Stelzer, B., Burwitz, V., Audard, M., et al. 2002, A\&A, 392, 585

Stelzer, B., Fernández, M., Costa, V. M., et al. 2003, A\&A, 411, 517 (Paper I)

Stepanov, A. V., Fürst, E., Krüger, A., et al. 1995, A\&A, 299, 739

Taylor, S. R. 1992, Solar System Evolution (Cambridge: Cambridge Univ. Press)

Tsuboi, Y., Imanishi, K., Koyama, K., Grosso, N., \& Montmerle, T. 2000, ApJ, 532, 1089

Valenti, J. A., Basri, G., \& Johns, C. M. 1993, AJ, 106, 2024

van den Oord, G. H. J., Doyle, J. G., Rodonò, M., et al. 1996, A\&A, 310, 908

Vilhu, O., Tsuru, T., Collier Cameron, A., et al. 1993, A\&A, 278, 467

Vrba, F. J., Herbst, W., \& Booth, J. F. 1988, AJ, 96, 1032

Welty, A. D., \& Ramsey, L. W. 1995, AJ, 110, 336

Wetherill, G. W., \& Stewart, G. R. 1993, Icarus, 106, 190

Wichmann, R., Bastian, U., Krautter, J., Jankovics, I., \& Rucinski, S. M. 1998, MNRAS, 301, L39 